CERN-TH.7178/94

\title{
Dilaton Production in String Cosmology
}

\author{
M.Gasperini \\ Dipartimento di Fisica Teorica, Via P.Giuria 1, 10125 Turin, Italy, \\ and INFN, Sezione di Torino, Turin, Italy \\ and \\ G.Veneziano \\ Theory Division, CERN, Geneva, Switzerland
}

\begin{abstract}
We consider the coupled evolution of density, (scalar) metric and dilaton perturbations in the transition from a "stringy" phase of growing curvature and gravitational coupling to the standard radiation-dominated cosmology. We show that dilaton production, with a spectrum tilted towards large frequencies, emerges as a general property of this scenario. We discuss the frame-independence of the dilaton spectrum and of the inflationary properties of the metric background by using, as model of source, a pressureless gas of weakly interacting strings, which is shown to provide an approximate but consistent solution to the full system of background equations and string equations of motion. We combine various cosmological bounds on a growing dilaton spectrum with the bound on the dilaton mass obtained from tests of the equivalence principle, and we find allowed windows compatible with a universe presently dominated by a relic background of dilatonic dark matter.
\end{abstract}

CERN-TH.7178/94

February 1994 


\section{Introduction and motivations.}

It is well known that fluctuations of the metric background are amplified in the context of inflationary cosmologies, and that the amplification of their transverse, traceless (spin 2) component can be interpreted as graviton production [1]. Models of the early universe based on the low-energy string effective action (what we shall refer to, for short, as "string cosmology"), are characterized by the additional presence of a dilaton background, $\phi(t)$. It is natural to expect an amplification of the fluctuations $\delta \phi \equiv \chi$ of the dilaton background - with corresponding dilaton production - to accompany that of the metric for a suitable time-evolution of the cosmological fields.

In this paper we discuss such a dilaton production in the context of the socalled "pre-big-bang' scenario [2], characterized by an accelerated evolution from a flat, cold and weakly coupled initial regime to a final hot, highly curved, strong coupling regime, marking the beginning of the standard "post-big-bang" decelerated FRW cosmology. With this background, the spectrum of the produced dilatons tends to grow with frequency, just like in the (previously discussed $[2,3]$ ) case of graviton production. As we shall see in Sec.3, the high frequency part of the spectral distribution, $\Omega_{\chi}$, of the dilaton energy density can be parameterized (in units of critical density $\rho_{c}=H^{2} / G=M_{p}^{2} H^{2}$ ) as

$$
\Omega_{\chi}(\omega, t)=\frac{\omega}{\rho_{c}} \frac{d \rho_{\chi}}{d \omega} \simeq G H_{1}^{2}\left(\frac{\omega}{\omega_{1}}\right)^{\delta}\left(\frac{H_{1}}{H}\right)^{2}\left(\frac{a_{1}}{a}\right)^{4}, \quad \delta>0, \quad \omega<\omega_{1}
$$

where $M_{p} \simeq 10^{19} \mathrm{GeV}$ is the Planck mass. Here $H_{1}$ is the curvature scale evaluated at the time $t_{1}$ marking the end of the inflationary epoch (which we assume to coincide with the beginning of radiation dominance); $\omega_{1}=H_{1} a_{1} / a$ is the maximum amplified proper frequency; $H=\dot{a} / a$, where $a$, as usual, is the scale factor of the background metric. The integral over $\omega$ is thus dominated by the highest frequency $\omega_{1}$

$$
\Omega_{\chi}(t)=\int^{\omega_{1}} \frac{d \omega}{\omega} \Omega_{\chi}(\omega, t) \simeq G H_{1}^{2}\left(\frac{H_{1}}{H}\right)^{2}\left(\frac{a_{1}}{a}\right)^{4}
$$

and the condition $\Omega_{\chi}<1$, required to avoid that the dilatons overclose the universe in the radiation-dominated era $\left(a \sim H^{-1 / 2}\right)$, yields the constraint $H_{1} \lesssim M_{p}$, already known $[2,3]$ from the graviton spectrum.

The produced dilatons, however, cannot be massless. Large distance dilaton couplings can be estimated [4] and turn out to be at least of gravitational strength. This violates the universality of gravity at low energy and, in particular, induces corrections to the effective Newton potential (in the static, weak-field limit), which 
may be reconciled with the present tests of the equivalence principle [5] only for a dilaton mass satisfying $[4,6]$ :

$$
m \gtrsim m_{0} \equiv 10^{-4} \mathrm{eV}
$$

The expression (1.2) is thus valid only until the energy density stays dominated by the relativistic modes, with $\omega(t)>m$. But, at the present time $t_{0}$ (with $\left.H_{0} \sim 10^{-61} M_{p}\right)$, the maximum frequency $\omega_{1}$ is

$$
\omega_{1}\left(t_{0}\right)=H_{1} \frac{a_{1}}{a_{0}} \simeq 10^{-4}\left(\frac{H_{1}}{M_{p}}\right)^{1 / 2} \mathrm{eV}
$$

As $H_{1}<M_{p}$, even the highest mode must then become non-relativistic before the present epoch, because of the constraint (1.3). At the scale $H\left(t_{m}\right)=m$ the modes with $\omega\left(t_{m}\right) \lesssim m$ begin to oscillate coherently, with frequency $m$, and when they are dominant the dilaton energy density becomes non-relativistic, with

$$
\begin{array}{cl}
\Omega_{\chi}(t) \simeq G m H_{1}\left(\frac{H_{1}}{H}\right)^{2}\left(\frac{m}{H_{1}}\right)^{\frac{\delta-1}{2}}\left(\frac{a_{1}}{a}\right)^{3}, & 0 \leq \delta \leq 1 \\
\Omega_{\chi}(t) \simeq G m H_{1}\left(\frac{H_{1}}{H}\right)^{2}\left(\frac{a_{1}}{a}\right)^{3}, & \delta \geq 1
\end{array}
$$

(the dependence on the spectral index $\delta$ disappears for fast enough growth of the spectrum, as shown in Sec.5). Such a fraction of critical density grows in time during the radiation era, while in the matter era $\left(a \sim H^{-2 / 3}\right)$ it becomes fixed at the maximum constant value

$$
\begin{array}{cc}
\Omega_{\chi} \simeq G m^{2}\left(\frac{H_{1}}{H_{2}}\right)^{1 / 2}\left(\frac{m}{H_{1}}\right)^{\frac{\delta-3}{2}}, & 0 \geq \delta \geq 1 \\
\Omega_{\chi} \simeq G m H_{1}\left(\frac{H_{1}}{H_{2}}\right)^{1 / 2}, & \delta \geq 1
\end{array}
$$

where $H_{2} \sim 10^{6} H_{0}$ is the curvature scale at the matter-radiation transition. The condition $\Omega_{\chi} \leq 1$ provides then, for any given inflation scale $H_{1}$, an upper limit for the dilaton mass,

$$
\begin{array}{lr}
m \lesssim\left(H_{2} M_{p}^{4} H_{1}^{\delta-4}\right)^{1 /(\delta+1)}, & 0 \leq \delta \leq 1 \\
m \lesssim H_{2}^{1 / 2} M_{p}^{2} H_{1}^{-3 / 2}, & \delta \geq 1
\end{array}
$$


valid for $m<H_{1}$ under the assumption that its life-time is sufficiently long to reach the matter-dominated era (if $m>H_{1}$ the dilaton must decay before becoming dominant with respect to the radiation, as we shall see in Sec.5, and the critical density bound cannot be applied).

In addition to the constraint (1.7), which is an unavoidable consequence of the quantum fluctuations of the dilaton background, one should also consider, in general, the constraints following from possible classical oscillations of the background around the minimum of the potential [7]. The initial amplitude of such oscillations is, however, to a large extent model-dependent and, as discussed in Sec.5, we shall work under the assumption that classical oscillations are negligible with respect to the quantum fluctuations amplified by the cosmological evolution. This assumption will give us the maximum allowed region in parameter space.

In the absence of classical oscillations, the upper limit on $m$ obtained from eq.(1.7), combined with the lower limit (1.3), define for each value of $\delta$ and $H_{1}$ an allowed window for the dilaton mass such that, near the upper end of the window, the produced dilatons can close the universe. Such a dilaton dominance, however, can last only until their energy density is dissipated into radiation, which occurs at a decay scale

$$
H_{d} \simeq \Gamma_{d} \simeq \frac{m^{3}}{M_{p}^{2}}
$$

We are thus led to the first interesting result of this analysis. If $\delta \gtrsim 0.1$, the upper limit (1.7) turns out to be larger than the lower bound (1.3) even for inflation scales $H_{1} \geq 10^{-5} M_{p}$. Moreover, always for $H_{1} \geq 10^{-5} M_{p}$, the largest value of $m$ allowed by eq.(1.7) is $m \simeq 100 \mathrm{MeV}$ (obtained for $\delta \geq 1$ ), and it implies $H_{d} \lesssim H_{0}$. This means that, for fast enough growing spectra, and "realistic" (at least in a string cosmology context) inflation scales $H_{1} \geq 10^{-5} M_{p}$, we can be left today with a background of relic dilatons possibly representing a significant fraction of the dark matter background [8]. The allowed ranges of $m$ corresponding to this interesting possibility lie around the upper limits given in (1.7) and thus depend on $\delta$ and $H_{1}$ in a complicated way. For $0.1 \lesssim \delta \lesssim 0.72$ the range of $H_{1}$ for which this possibility can be realized is given by

$$
H_{1} \lesssim 10^{-(23-32 \delta) /(4-\delta)} M_{p}
$$

while for $\delta>0.72$ values of $H_{1} / M_{p}$ up to 1 are possible (the case $\delta \geq 1$ is illustrated in Fig.1). The lower bound on $\delta$ is imposed by the simultaneous requirements $H_{1} \geq 10^{-5} M_{p}$ and $m \geq m_{0}$, together with eq.(1.7). As $H_{1} / M_{p}$ is varied between 
$10^{-5}$ and 1 the corresponding dilaton mass vary over the whole domain from $10^{-4} \mathrm{eV}$ to $100 \mathrm{MeV}$. For lower spectral slopes $(\delta<0.1)$, the present existence of a dominant dilaton background becomes possible only for (unrealistically) low inflation scales, as first pointed out in Ref.[9] for the case of scalar perturbations with a flat $(\delta=0)$ spectrum. Note that, according to eq.(1.9), a final inflation scale $H_{1}$ exactly coinciding with the string scale itself, $\sim 10^{-1} M_{p}$, would be compatible with a present light dilaton dominance only for $\delta \gtrsim 0.61$.

The inflation scale $H_{1}$ determines also the amplitude of scalar perturbations $\psi$ of the metric itself, and it is thus constrained by the scalar contribution to the CMBR anisotropies (the contribution of tensor perturbations turns out to be negligible in our context, as their spectrum grows very fast with frequency $[2,3])$. The behavior of the scalar perturbation spectrum, as we shall see, depends in general on the adopted model of matter sources and background evolution, and it is fair to say that our present knowledge of the details of the stringy pre-big-bang phase is to poor to make stringent predictions on the exact value of the spectral index $\delta$. On the other hand, fortunately enough, the properties of a massive dilaton background are only weakly dependent on the value of the spectral index for $\delta>0$, and rapidly become spectrum-independent as soon as $\delta \geq 1$. The particular example chosen in this paper (see Sec.4) to discuss dilaton production, namely a three-dimensional, isotropic, dilaton-dominated background with negligible matter sources, gives the same spectrum (very fast growing, $\delta=3$ ), for scalar $(\psi$ ), dilaton $(\chi)$ and tensor $\left(h_{\mu \nu}\right)$ perturbations. However, such an example is chosen here for simplicity reasons only, in order to develop a first qualitative sketch of the scenario associated with dilaton production, and it should not be taken as particularly indicative for what regards the spectral properties of the metric perturbations. For a phenomenological discussion it is better to leave open the possibility of different spectra for $\psi$ and $\chi$ (possibility which is in general allowed in this context, as we shall see in Sec.3), and to parameterize the scalar (metric) energy density as

$$
\Omega_{\psi}(\omega, t) \simeq G H_{1}^{2}\left(\frac{\omega}{\omega_{1}}\right)^{n-1}\left(\frac{H_{1}}{H}\right)^{2}\left(\frac{a_{1}}{a}\right)^{4}
$$

with $n-1$ in general different from $\delta$.

The interesting question to ask at this point is whether, in the same range of $H_{1}$ which we believe to be realistic, it is possible to produce enough dilatons to close the present universe and, at the same time, to generate scalar perturbations with a spectrum consistent with the anisotropy observed by COBE [10]. This 
amounts to require

$$
10^{-5} \lesssim \frac{H_{1}}{M_{p}} \lesssim 1, \quad \Omega_{\chi}\left(t_{0}\right) \simeq 1, \quad \Omega_{\psi}\left(\omega_{0}, t_{2}\right) \simeq G H_{1}^{2}\left(\frac{\omega_{0}}{\omega_{1}}\right)^{n-1} \simeq 10^{-10}
$$

where $\omega_{0}=H_{0}$ is the minimum amplified frequency corresponding to a wave crossing today the Hubble radius, and $t_{2}$ is the time of matter-radiation equilibrium, nearly coincident with the time of recombination.

The answer, perhaps surprisingly, is yes: the last requirement of eq.(1.11) is compatible with eq.(1.9), for the same range of $H_{1}$, provided the scalar spectrum is also growing, with

$$
1 \lesssim n \lesssim 1.34
$$

This allowed range of $n$ is well contained in the range of the spectral index originally determined by COBE [10], $n=1 \pm 0.5$, and is also consistent with the new recent fit [11] which gives $n=1.5 \pm 0.5$. It may be interesting to recall, in this context, that growing scalar perturbations, with $n \simeq 1.25$, are also required for a simultaneous fit of the COBE anisotropies and of the observed bulk motion and large voids structures on a $50 M p c$ scale [12]. Growing scalar spectra can be obtained in the "hybrid inflation" model proposed by Linde [13] and recently generalized to the class of "false vacuum inflation" [14] (see also [15]). Note also that the condition (1.12) would not be incompatible with the lower bound on $\delta$ required for a present dominant dilaton background (according to eq.(1.9)), even in the case of equal scalar and dilaton spectrum, $\delta=n-1$.

Concluding this qualitative analysis, we can say that the possibility of producing a dilaton background which saturates the closure density, together with scalar perturbations which provide the observed cosmic anisotropies, seems to be naturally associated with a growing dilaton spectrum, $\delta>0$. The fact that such a spectrum is typical of string-based pre-big-bang models represents, in our opinion, an interesting aspect of such models, and motivates the study of dilaton production in the string cosmology scenario. A requirement analogous to eq.(1.11), formulated however in the context of extended inflation models where the fluctuation spectrum of the Brans-Dicke scalar is not growing, may be satisfied [16] only for a reheating temperature $T_{r}<10^{13} \mathrm{GeV}$, namely for very low scales $H_{1} \simeq T_{r}^{2} / M_{p} \leq 10^{-12} M_{p}$. We note, finally, that the possibility of inflationary production of massless scalar particles, associated with excitations of the Brans-Dicke field, was also pointed out in Ref.[17], and previously discussed in Ref.[18] for the massive case (with $m<H_{1}$ ), but always in the context of exponential inflation, which is not the 
natural inflationary background corresponding to the low energy string effective action.

The paper is organized as follows.

In Sec.2 we present the general exact solutions (for space-independent fields) of the system of background field equations, including classical string sources, following from the tree-level string effective action at lowest order in $\alpha^{\prime}$. The explicit form of the solution is displayed, in particular, for a perfect fluid model of sources, in $D=d+1$ dimensions, for any given equation of state. The low curvature and large curvature limit of such solutions are given both in the Brans-Dicke and in the conformally related Einstein frame. In Sec.3 we derive the coupled system of scalar (metric plus dilaton) perturbation equations, including the perturbations of the matter sources in the perfect fluid form. Such equations are applied to compute the scalar perturbation spectrum for a specific case of background evolution motivated by a model of sources (presented in Sec.4) in which the dominant form of matter is a sufficiently diluted, non-interacting gas of large macroscopic strings. The background describes a phase of growing curvature and accelerated kinematic (of the pre-big-bang type), which is expected to evolve towards the standard, radiation-dominated cosmology. The frame-independence of the inflationary properties of such background is also discussed in Sec.4. The corresponding spectrum of the produced dilatons is discussed in Sec.5, where it is shown that, because of its fast growth with frequency, the phenomenological constraints leave open a window compatible with the possible dominance of not yet decayed dilatons (in the hypothesis of negligible classical oscillations of the dilaton background). The main results of this paper are finally summarized and briefly discussed in Sec.6.

\section{General solution of the background field equations.}

We will assume the evolution of the Universe to be described at curvatures below the string/Planck scale by the equations

$$
\begin{gathered}
R_{\mu}{ }^{\nu}+\nabla_{\mu} \nabla^{\nu} \phi-\frac{1}{2} \delta_{\mu}{ }^{\nu} \frac{\partial V}{\partial \phi}-\frac{1}{4} H_{\mu \alpha \beta} H^{\nu \alpha \beta}=8 \pi G_{D} e^{\phi} T_{\mu}^{\nu} \\
R-\left(\nabla_{\mu} \phi\right)^{2}+2 \nabla_{\mu} \nabla^{\mu} \phi+V-\frac{\partial V}{\partial \phi}-\frac{1}{12} H_{\mu \nu \alpha} H^{\mu \nu \alpha}=0 \\
\partial_{\nu}\left(\sqrt{|g|} e^{-\phi} H^{\mu \alpha \beta}\right)=0
\end{gathered}
$$


Such system of equations follows from the low-energy ( $D$-dimensional) effective action of closed (super)string theory [19],

$$
S=-\frac{1}{16 \pi G_{D}} \int d^{D} x \sqrt{|g|} e^{-\phi}\left[R+\partial_{\mu} \phi \partial^{\mu} \phi-\frac{1}{12} H_{\mu \nu \alpha} H^{\mu \nu \alpha}+V(\phi)\right]+S_{M}
$$

Here $\phi$ is the dilaton field, and $H_{\mu \nu \alpha}$ the field strength of the two-index antisymmetric (torsion) tensor $B_{\mu \nu}=-B_{\nu \mu}$. We have included a possible dilaton potential, $V(\phi)$, and also a possible phenomenological contribution of the matter sources represented by the action $S_{M}$, whose metric variation produces the stress tensor $T_{\mu \nu}$.

We shall consider, in this paper, homogeneous backgrounds which are independent of all spacelike coordinates (Bianchi I type, with $d$ Abelian isometries), and for which a synchronous frame exists where $g_{00}=1, g_{0 i}=0=B_{0 i}$ (conventions: $\mu, \nu=0,1, \ldots . D=d+1 ; i, j=1,2, \ldots . d)$. We shall assume, moreover, that the action $S_{M}$ describes "bulk" string matter, satisfying the classical string equations of motion in the given background. At tree level $V$ is a constant. In terms of the "shifted dilaton"

$$
\bar{\phi}=\phi-\frac{1}{2} \ln \left|\operatorname{det}\left(g_{\mu \nu}\right)\right|
$$

the field equations $(2.1)-(2.3)$ can be written in matrix form as [20]

$$
\begin{gathered}
\dot{\bar{\phi}}^{2}-2 \ddot{\bar{\phi}}-\frac{1}{8} \operatorname{Tr}(\dot{M} \eta)^{2}-V=0 \\
\dot{\bar{\phi}}^{2}+\frac{1}{8} \operatorname{Tr}(\dot{M} \eta)^{2}-V=\bar{\rho} e^{\bar{\phi}} \\
\frac{d}{d t}\left(e^{-\bar{\phi}} M \eta \dot{M}\right)=\bar{T}
\end{gathered}
$$

(a dot denotes differentiation with respect to the cosmic time $t$, and we have used units in which $8 \pi G_{D}=1$, so that $\bar{\rho}$ and $\bar{T}$ have both dimensions $L^{-2}$ ). Here $M$ is a $2 d \times 2 d$ matrix,

$$
M=\left(\begin{array}{cc}
G^{-1} & -G^{-1} B \\
B G^{-1} & G-B G^{-1} B
\end{array}\right)
$$

where $G$ and $B$ are matrix representation of the $d \times d$ spatial part of the metric $\left(g_{i j}\right)$ and of the antisymmetric tensor $\left(B_{i j}\right)$, in the basis in which the $O(d, d)$ metric $\eta$ is in off-diagonal form,

$$
\eta=\left(\begin{array}{ll}
0 & I \\
I & 0
\end{array}\right)
$$


( $I$ is the unit $d \times d$ matrix). $\bar{T}$ is another $2 d \times 2 d$ matrix representing the spatial part of the string stress tensor [20] (including the possible contribution of an antisymmetric current density, source of torsion). Finally $\bar{\rho}$ is related to the energy density $\rho=T_{0}{ }^{0}$ by

$$
\bar{\rho}=\rho \sqrt{\left|\operatorname{det}\left(g_{\mu \nu}\right)\right|}
$$

The three equations (2.6)-(2.8) correspond, respectively, to to the dilaton equation (2.2) and to the time and space part of eqs.(2.1), (2.3) for the homogeneous background that we have considered. Their combination provides the covariant conservation equation for the source energy density, which can be written in compact form as [20]

$$
\dot{\bar{\rho}}+\frac{1}{4} \operatorname{Tr}(\bar{T} \eta M \eta \dot{M} \eta)=0
$$

The set of equations (2.6)-(2.8), (2.12) is explicitly covariant under the global $O(d, d)$ transformation $[21,20]$

$$
\bar{\phi} \rightarrow \bar{\phi}, \quad \bar{\rho} \rightarrow \bar{\rho}, \quad M \rightarrow \Lambda^{T} M \Lambda, \quad \bar{T} \rightarrow \Lambda^{T} \bar{T} \Lambda
$$

where $\Lambda$ is an $O(d, d)$ constant matrix satisfying

$$
\Lambda^{T} \eta \Lambda=\eta
$$

For a suitable class of dilaton potentials such a system can be solved by quadratures, following the method presented in Ref.[2].

Here we shall concentrate, in particular, on the case $V=0$, corresponding to strings in critical space-time dimensions (which does not exclude, however, a description of $d=3$ evolution in case we add the right number of spectator dimensions in order to compensate the central charge deficit). We introduce a suitable (dimensionless) time coordinate $x$, such that

$$
\bar{\rho}=\frac{1}{L} \frac{d x}{d t}
$$

( $L$ is a constant with dimensions of length), and we define

$$
\Gamma=\int^{x} \frac{\bar{T}}{\bar{\rho}} d x^{\prime}
$$

The equations (2.6)-(2.8) can then be integrated a first time, with the help of eq.(2.16) and of the $O(d, d)$ identity

$$
(M \eta \dot{M} \eta)^{2}=-(\dot{M} \eta)^{2},
$$


to give $[2]$

$$
\begin{gathered}
\bar{\rho}=\frac{e^{\bar{\phi}}}{4 L^{2}} D \\
\bar{\phi}^{\prime}=-\frac{2}{D}\left(x+x_{0}\right) \\
M \eta M^{\prime}=\frac{4 \Gamma}{D}
\end{gathered}
$$

where

$$
D=\left(x+x_{0}\right)^{2}-\frac{1}{2} \operatorname{Tr}(\Gamma \eta)^{2}
$$

(a prime denotes differentiation with respect to $x$, and $x_{0}$ is an integration constant).

By exploiting the fact that $M$ is a symmetric $O(d, d)$ matrix, $M \eta M=\eta$, and that, because of the definition of $\bar{T}$ (see Ref.[20])

$$
M \eta \Gamma=-\Gamma \eta M
$$

eqs.(2.19), (2.20) can be integrated a second time to give

$$
\begin{gathered}
\bar{\phi}(x)=\phi_{0}-2 \int \frac{d x}{D}\left(x+x_{0}\right) \\
M(x)=P_{x} \exp \left(-4 \int \frac{d x}{D} \Gamma \eta\right) M_{0}
\end{gathered}
$$

where $\phi_{0}$ and $M_{0}$ are integration constants $\left(M_{0}\right.$ is a symmetric $O(d, d)$ matrix), and $P_{x}$ denotes $x$ - ordering of the exponential. For any given "equation of state", providing a relation $\bar{T}=\bar{T}(\bar{\rho})$ between the spatial part of the stress tensor of the sources and their energy density, eqs.(2.23) and (2.24), together with (2.18), represent the general exact solution of the system of string cosmology equations, for space-independent background fields and vanishing dilaton potential.

Such a solution presents in general singularities, for the curvature and the effective coupling constant $e^{\phi}$, occurring in correspondence of the zero of $D(x)$. It is important to stress that, near the singularity, the contribution of the matter sources becomes negligible with respect to the curvature terms in the field equations (just like in general relativity, in the case of Kasner's anisotropic solution).

The relative importance of the source term is measured indeed by the ratio (see for instance eq.(2.7))

$$
\Omega(x)=-\frac{8 \bar{\rho} e^{\bar{\phi}}}{(d-1) \operatorname{Tr}(\dot{M} \eta)^{2}}
$$


(we have normalized $\Omega$ in such a way that it reduces to the usual expression for the effective energy density in critical units, $\Omega=\rho / \rho_{c}$, when the dilaton is constant and the metric isotropic). According to eqs.(2.18), (2.20)

$$
M \eta \dot{M}=\frac{e^{\bar{\phi}}}{L} \Gamma
$$

so that, by exploiting the $O(d, d)$ properties of $M$,

$$
\operatorname{Tr}(\dot{M} \eta)^{2}=-\frac{e^{2 \bar{\phi}}}{L^{2}} \operatorname{Tr}(\Gamma \eta)^{2}
$$

Therefore

$$
\Omega=\frac{2}{d-1} \frac{D}{\operatorname{Tr}(\Gamma \eta)^{2}}
$$

goes to zero at the singularity $(D \rightarrow 0)$.

In this limit, the matter contribution becomes negligible and the general solution presented here reduces to the $V=0$ case of the general vacuum solution of the string cosmology equations [22]. Denoting indeed by $t_{c}$ a singular point such that $D\left(t_{c}\right)=0, \Gamma\left(t_{c}\right) \neq 0$, from eq.(2.26) we have, near this point,

$$
M \eta \dot{M} e^{-\bar{\phi}}=A
$$

where the constant matrix $A$ satisfies

$$
A=\frac{\Gamma\left(t_{c}\right)}{L}=-A^{T}, \quad M \eta A+A \eta M=0
$$

because of the property (2.22) of $\Gamma$. Moreover, from (2.18) and (2.19)

$$
\dot{\bar{\phi}}^{2}=\frac{e^{2 \bar{\phi}}}{4 L^{2}}\left(x+x_{0}\right)^{2}
$$

so that, by using (2.27),

$$
\dot{\bar{\phi}}^{2}+\frac{1}{8} \operatorname{Tr}(\dot{M} \eta)^{2}=\frac{e^{2 \bar{\phi}}}{4 L^{2}} D\left(t_{c}\right)=0
$$

Eqs.(2.29) and (2.32) correspond exactly to the equations defining the general vacuum solution of Ref.[22], for the case of vanishing dilaton potential.

Consider now the particular case in which $B_{\mu \nu}=0$, and we are in a diagonal, but not necessarily isotropic, Bianchi-I type metric background,

$$
g_{00}=1, \quad g_{i j}=-a_{i}^{2}(t) \delta_{i j}
$$


(this is the background that will we used here to discuss dilaton production). The matter sources can be represented in the perfect fluid form, but with anisotropic pressure,

$$
T_{0}^{0}=\rho, \quad T_{i}^{j}=-p_{i} \delta_{i}{ }^{j}, \quad p_{i} / \rho=\gamma_{i}=\text { const }
$$

In this case we obtain, from the previous definitions,

$$
\begin{gathered}
M \eta M^{\prime}=2\left(\begin{array}{cc}
0 & \frac{a_{i}^{\prime}}{a_{i}} \delta_{i j} \\
-\frac{a_{i}^{\prime}}{a_{i}} \delta_{i j} & 0
\end{array}\right), \quad \bar{T}=\left(\begin{array}{cc}
0 & \bar{p}_{i} \delta_{i j} \\
-\bar{p}_{i} \delta_{i j} & 0
\end{array}\right) \\
\gamma=\left(\begin{array}{cc}
0 & \Gamma_{i} \delta_{i j} \\
-\Gamma_{i} \delta_{i j} & 0
\end{array}\right), \quad \Gamma_{i}=\gamma_{i} x+x_{i} \\
D=\left(x+x_{0}\right)^{2}-\sum_{i}\left(\gamma_{i} x+x_{i}\right)^{2}=\alpha\left(x-x_{+}\right)\left(x-x_{-}\right)
\end{gathered}
$$

where

$$
\begin{gathered}
\bar{p}_{i}=p_{i} \sqrt{|g|}, \quad \alpha=1-\sum_{i} \gamma_{i}^{2} \\
x_{ \pm}=\frac{1}{\alpha}\left\{\sum_{i} \gamma_{i} x_{i}-x_{0} \pm\left[\left(\sum_{i} \gamma_{i} x_{i}-x_{0}\right)^{2}+\alpha\left(\sum_{i} x_{i}^{2}-x_{0}^{2}\right)\right]^{1 / 2}\right\}
\end{gathered}
$$

and $x_{i}, x_{0}$ are integration constants. The general solution $(2.18),(2.23),(2.24)$ becomes explicitly [23]

$$
\begin{gathered}
a_{i}=a_{0 i}\left|\left(x-x_{+}\right)\left(x-x_{-}\right)\right|^{\gamma_{i} / \alpha}\left|\frac{x-x_{+}}{x-x_{-}}\right|^{\alpha_{i}} \\
e^{\bar{\phi}}=e^{\phi_{0}}\left|\left(x-x_{+}\right)\left(x-x_{-}\right)\right|^{-1 / \alpha}\left|\frac{x-x_{+}}{x-x_{-}}\right|^{-\sigma} \\
\bar{\rho}=\frac{\alpha}{4 L^{2}} e^{\phi_{0}}\left|\left(x-x_{+}\right)\left(x-x_{-}\right)\right|^{(\alpha-1) / \alpha}\left|\frac{x-x_{+}}{x-x_{-}}\right|^{-\sigma}
\end{gathered}
$$

where

$$
\sigma=\sum_{i} \alpha_{i} \gamma_{i}, \quad \alpha_{i}=\frac{\alpha x_{i}+\gamma_{i}\left(\sum_{i} \gamma_{i} x_{i}-x_{0}\right)}{\alpha\left[\left(\sum_{i} \gamma_{i} x_{i}-x_{0}\right)^{2}+\alpha\left(\sum_{i} x_{i}^{2}-x_{0}^{2}\right)\right]^{1 / 2}}
$$

and $a_{i 0}, \phi_{0}$ are additional integration constants.

This solution has two curvature singularities at $x=x_{ \pm}$. Near the singularity, the presence of matter becomes negligible,

$$
\Omega(x)=\frac{\alpha\left(x-x_{+}\right)\left(x-x_{-}\right)}{(d-1) \sum_{i}\left(\gamma_{i} x+x_{i}\right)^{2}} \rightarrow 0
$$


and one recovers the anisotropic vacuum solution of string cosmology in critical dimensions $[24,25]$. Indeed, for $x \rightarrow x_{ \pm}$, one has $|x| \sim|t|^{\alpha /\left(1 \pm \alpha \sum \alpha_{i} \gamma_{i}\right)}$, and the solution behaves like

$$
a_{i}(t) \sim\left|t-t_{ \pm}\right|^{\beta_{i}^{ \pm}}, \quad \bar{\phi} \sim-\ln \left|t-t_{ \pm}\right|
$$

where

$$
\beta_{i}^{ \pm}=\frac{x_{i} \pm \gamma_{i} x_{ \pm}}{x_{0}+x_{ \pm}} \quad, \quad \sum_{i}\left(\beta_{i}^{ \pm}\right)^{2}=1 .
$$

In the large $|x|$ (small curvature) limit, on the contrary, the relation between $x$ and cosmic time is $|x| \simeq|t|^{\alpha /(2-\alpha)}$, and the solution (2.37)-(2.39) behaves like (for $|x| \rightarrow \pm \infty$ )

$$
\begin{aligned}
& a_{i}(t) \sim|t|^{2 \gamma_{i} /\left(1+\sum \gamma_{i}^{2}\right)}, \quad \bar{\phi} \sim-\frac{2}{1+\sum \gamma_{i}^{2}} \ln |t| \\
& \phi \sim 2 \frac{\sum \gamma_{i}-1}{1+\sum \gamma_{i}^{2}} \ln |t|, \quad \bar{\rho} \sim|t|^{-2 \sum \gamma_{i}^{2} /\left(1+\sum \gamma_{i}^{2}\right)}
\end{aligned}
$$

The critical density parameter, in this limit, goes to a constant

$$
\Omega_{\infty}=\frac{1-\sum_{i} \gamma_{i}^{2}}{(d-1) \sum_{i} \gamma_{i}^{2}}
$$

which is obviously $\Omega_{\infty}=1$ for an isotropic, radiation-dominated background with $\gamma_{i}=1 / d$.

It is interesting to point out that, for any solution $a_{i}, \phi$ corresponding to a given set of equations of state, $p_{i}=\gamma_{i} \rho$, there are the corresponding "dual" solutions obtained through the reflection $\gamma_{i} \rightarrow-\gamma_{i}$, which leads to $a_{i}\left(\gamma_{i}\right) \rightarrow a_{i}\left(-\gamma_{i}\right)=$ $a_{i}^{-1}\left(\gamma_{i}\right)$, preserving however the values of $\bar{\phi}$ and $\bar{\rho}$ (scale-factor duality [25,26]). Such a duality transformation, combined with the time- inversion $t \rightarrow-t$, transforms any given metric describing (for $\gamma_{i}>0$ ) decelerated expansion with decreasing curvature, $\ddot{a}_{i}<0, H_{i}>0, \dot{H}_{i}<0$, into a new solution describing (for $\gamma_{i}<0$ ) a superinflationary expansion with increasing curvature, $\ddot{a}_{i}>0, H_{i}>0, \dot{H}_{i}>0$ (see also [2]).

It is convenient, for later use, to write down explicitly the isotropic version of the asymptotic backgrounds (2.42) and (2.44), as a function of the cosmic time $t$ and conformal time $\eta$ such that $d t=a d \eta$. In the $(d+1)$-dimensional isotropic case, the small curvature limit (2.44) becomes, in cosmic time,

$$
a(t) \sim|t|^{2 \gamma /\left(1+d \gamma^{2}\right)}, \quad \phi \sim \frac{d \gamma-1}{\gamma} \ln a, \quad \rho \sim a^{-d(\gamma+1)}
$$


while in terms of $\eta$ we have

$$
a(\eta) \sim|\eta|^{2 \gamma /\left(1-2 \gamma+d \gamma^{2}\right)}
$$

The vacuum, dilaton-dominated limit (2.42) becomes, in the isotropic case,

$$
a_{\mp}(t) \sim|t|^{\mp 1 / \sqrt{d}}, \quad \phi_{\mp} \sim \sqrt{d}(\sqrt{d} \pm 1) \ln a
$$

and, in conformal time,

$$
a_{\mp}(\eta) \sim|\eta|^{\mp 1 /(\sqrt{d} \pm 1)}
$$

Note that for $\gamma=1 / d$, and $t \rightarrow+\infty$, eq.(2.46) describes the standard, radiationdominated cosmology with $\phi=$ const; the dual case, $\gamma=-1 / d$ and $t \rightarrow 0$, with $t<0$, describes instead a typical pre-big-bang configuration [2], with a superinflationary expansion driven by a perfect gas of stretched strings [27]. The dual solution obtained through a more general $O(d, d)$ transformation, applied to the radiation case, corresponds to a non-diagonal metric and an effective viscosity in the source stress tensor, and has been discussed in Ref.[20].

We note, finally, that the solution presented in this section is given explicitly in the Brans-Dicke (BD) frame, whose metric coincides with the $\sigma$-model metric to which strings are directly coupled. The passage to the Einstein (E) frame, defined as the frame in which the graviton and dilaton kinetic terms are diagonalized, and the action takes the canonical form

$$
S_{E}=\frac{1}{16 \pi G_{D}} \int d^{D} x \sqrt{\left|g_{E}\right|}\left[-R\left(g_{\mu \nu}^{E}\right)+\frac{1}{2} g_{E}^{\mu \nu} \partial_{\mu} \phi_{E} \partial_{\nu} \phi_{E}\right]
$$

is obtained through the conformal rescaling

$$
g_{\mu \nu}^{E}=g_{\mu \nu} e^{-2 \phi /(d-1)}, \quad \phi_{E}=\sqrt{\frac{2}{d-1}} \phi
$$

The E-transformed scale-factor, $a_{E}$, and the cosmic time coordinate, $t_{E}$, are thus related to the original $\mathrm{BD}$ ones by

$$
a_{E}=a e^{-\phi /(d-1)}, \quad d t_{E}=d t e^{-\phi /(d-1)}
$$

The asymptotic limit (2.46) of the previous general solution thus becomes, in the E frame,

$$
a_{E}\left(t_{E}\right) \sim\left|t_{E}\right|^{\beta}, \quad \phi_{E} \sim \sqrt{\frac{2}{d-1}} \frac{(d-1)(1-d \gamma)}{\gamma-1} \ln a_{E}
$$




$$
\rho_{E} \sim a_{E}^{-2 / \beta}, \quad \beta=\frac{2(1-\gamma)}{(d-1)\left(1+d \gamma^{2}\right)-2(d \gamma-1)}
$$

where $\rho_{E}$ is conformally related to the BD energy density $\rho$ by

$$
\rho_{E}=\rho \frac{\sqrt{|g|}}{\sqrt{\left|g_{E}\right|}}=\rho e^{\phi(d+1) /(d-1)}
$$

In conformal time,

$$
a_{E}(\eta) \sim|\eta|^{-2(\gamma-1) /(d-1)\left(1-2 \gamma+d \gamma^{2}\right)}
$$

(note that the conformal time coordinate is the same in the $\mathrm{E}$ and $\mathrm{BD}$ frame,

$$
d \eta_{E}=\frac{d t_{E}}{a_{E}\left(t_{E}\right)}=\frac{d t}{a(t)}=d \eta
$$

because of eq.(2.52)). The high curvature limit (2.48) becomes, in the E frame,

$$
a_{\mp}^{E}\left(t_{E}\right) \sim\left|t_{E}\right|^{1 / d}, \quad \quad \phi_{\mp}^{E} \sim \mp \sqrt{2 d(d-1)} \ln a_{E}
$$

and, in conformal time,

$$
a_{\mp}^{E}\left(\eta_{E}\right) \sim|\eta|^{1 /(d-1)}
$$

It should be stressed that the radiation-dominated solution, with $\gamma=1 / d$, $\phi=$ const, is obviously the same in both frames, see eqs.(2.53) and (2.48). We note also that in the vacuum, dilaton-dominated case, the duality transformation which is represented in BD frame by the inversion of the scale factor, with related dilaton shift,

$$
a_{ \pm} \rightarrow a_{\mp}=a_{ \pm}^{-1}, \quad \phi_{ \pm} \rightarrow \phi_{\mp}=\phi_{ \pm}-2 d \ln a_{ \pm}
$$

in the E frame becomes a transformation between the weak coupling and the strong coupling regime,

$$
\phi_{ \pm}^{E} \rightarrow \phi_{\mp}^{E}=-\phi_{ \pm}^{E}
$$

without changing the metric background.

Concluding this section, we want to stress that the solutions discussed so far describe the situation in which the dilaton potential can be neglected, namely the background evolution at early enough times when the effective coupling $e^{\phi}$ is small enough. Indeed, because of non- renormalization theorems, the potential is expected to appear at the non-perturbative level only, and has to be extremely small $(V(\phi) \sim \exp [-\exp (\phi)])$ in the weak coupling regime. At later times, and large couplings, the main effect of the dilaton potential will be taken into account 
in the form of a dilaton mass term (see Sections 3,5), which freezes the Newton constant at its present value.

\section{Scalar perturbations with dilaton and perfect fluid sources.}

In order to obtain the equations governing the classical evolution of scalar perturbations, we choose to work in the Einstein frame, where the explicit form of the equations is simpler. This is a legitimate choice since, as we shall see at the end of this Section, the scalar perturbation spectrum, just like the graviton spectrum [23], is the same in the Einstein and Brans-Dicke frames.

In the E frame, the background field equations (with $B_{\mu \nu}=0$ but with a non-vanishing dilaton potential $V$ ) take the form

$$
\begin{gathered}
2 R_{\mu}^{\nu}-\delta_{\mu}^{\nu} R=\partial_{\mu} \phi \partial^{\nu} \phi+\delta_{\mu}^{\nu}\left[V-\frac{1}{2}(\nabla \phi)^{2}\right]+T_{\mu}{ }^{\nu} \\
\nabla_{\mu} \nabla^{\mu} \phi+\frac{\partial V}{\partial \phi}+c T=0
\end{gathered}
$$

where $c=\sqrt{2 /(d-1)}$ (the Einstein frame index, E, will be omitted throughout this Section). The coupling of the dilaton to the stress tensor of the matter sources is fixed by the conformal rescaling $(2.51),(2.54)$. We start, for simplicity, with a $(d+1)$-dimensional isotropic background, with perfect fluid sources,

$$
\begin{array}{cc}
g_{\mu \nu}=\operatorname{diag}\left(1,-a^{2} \delta_{i j}\right), & \phi=\phi(t) \\
T_{\mu}{ }^{\nu}=(\rho+p) u_{\mu} u^{\nu}-p \delta_{\mu}^{\nu}, & u^{\mu}=\delta_{0}^{\mu}
\end{array}
$$

and we consider the pure scalar part of the metric perturbations, $\delta g_{\mu \nu} \equiv h_{\mu \nu}$, together with the perturbations of the dilaton background, $\delta \phi \equiv \chi$, and of the matter sources, $\delta \rho, \delta p, \delta u^{\mu}$ (in the linear approximation scalar, vector and tensor perturbations are decoupled, and evolve independently). We use here for the metric the Bardeen variables $\Phi, \psi$, that are invariant under those infinitesimal coordinate transformations which preserve the scalar nature of the fluctuations [28-30]. In the longitudinal gauge we have thus the first-order expressions [30]

$$
\begin{array}{cc}
h_{00}=2 \Phi=h^{00}, & h_{0 i}=0 \\
h_{i j}=2 a^{2} \psi \delta_{i j}, & h^{i j}=\frac{2}{a^{2}} \psi \delta^{i j}
\end{array}
$$




$$
\delta T_{0}{ }^{0}=\delta \rho, \quad \delta T_{i}{ }^{j}=-\delta_{i}^{j} \delta p, \quad \delta T_{i}{ }^{0}=\frac{\rho+p}{a} \delta u_{i}
$$

These are to be inserted into the first order perturbation of the Einstein equations $(3.1)$,

$$
\begin{gathered}
-2 h^{\nu \alpha} R_{\mu \alpha}+2 g^{\nu \alpha} \delta R_{\mu \alpha}-\delta_{\mu}^{\nu}\left(g^{\alpha \beta} \delta R_{\alpha \beta}-h^{\alpha \beta} R_{\alpha \beta}\right)= \\
=-h^{\nu \alpha} \partial_{\mu} \phi \partial_{\alpha} \phi+\partial_{\mu} \chi \partial^{\nu} \phi+\partial_{\mu} \phi \partial^{\nu} \chi+\delta_{\mu}^{\nu} \frac{\partial V}{\partial \phi} \chi- \\
-\frac{1}{2} \delta_{\mu}^{\nu}\left(2 g^{\alpha \beta} \partial_{\alpha} \phi \partial_{\beta} \chi-h^{\alpha \beta} \partial_{\alpha} \phi \partial_{\beta} \phi\right)+\delta T_{\mu}{ }^{\nu}
\end{gathered}
$$

and of the dilaton equation $(3.2)$

$$
-h^{\alpha \beta} \nabla_{\alpha} \nabla_{\beta} \phi+\nabla_{\mu} \nabla^{\mu} \chi-g^{\alpha \beta}\left(\delta \Gamma_{\alpha \beta}^{\mu}\right) \partial_{\mu} \phi+\frac{\partial^{2} V}{\partial \phi^{2}} \chi+\frac{c}{2}(\delta \rho-d \delta p)=0
$$

Here the covariant derivatives are to be performed with respect to the background metric $g_{\mu \nu}$, and $\delta R_{\mu \nu}, \delta \Gamma$ are to be computed to first order in $h_{\mu \nu}$.

By using the background field equations, the $(i, j)$ component of eq.(3.5), with $i \neq j$, gives

$$
\Phi=(d-2) \psi
$$

which allows us to eliminate everywhere one of the two Bardeen's variables. The $(i, 0)$ component gives a constraint which can be written, in terms of the conformal time $\eta$, as

$$
\partial_{i}\left\{2(d-1)\left[\frac{a^{\prime}}{a}(d-2) \psi+\psi^{\prime}\right]-\chi \phi^{\prime}\right\}=(\rho+p) a \delta u_{i}
$$

(a prime denotes differentiation with respect to $\eta)$. The $(0,0)$ component provides an expression for the density perturbation $\delta \rho$ in terms of the scalar variables $\psi$ and $\chi$,

$$
\begin{gathered}
\nabla^{2} \psi-d \frac{a^{\prime}}{a} \psi^{\prime}-\left[d(d-2)\left(\frac{a^{\prime}}{a}\right)^{2}-\frac{d-2}{2(d-1)} \phi^{\prime 2}\right] \psi= \\
=\frac{1}{2(d-1)}\left(\phi^{\prime} \chi^{\prime}+\frac{\partial V}{\partial \phi} a^{2} \chi+a^{2} \delta \rho\right)
\end{gathered}
$$

Finally, the $(i, i)$ component of eq.(3.5) and the perturbed dilaton equation (3.6) give, respectively,

$$
\begin{aligned}
\psi^{\prime \prime}+(2 d-3) \frac{a^{\prime}}{a} \psi^{\prime} & +\left\{(d-2)\left[2 \frac{a^{\prime \prime}}{a}+(d-4)\left(\frac{a^{\prime}}{a}\right)^{2}\right]+\frac{d-2}{2(d-1)} \phi^{\prime 2}\right\} \psi= \\
& =\frac{1}{2(d-1)}\left(\phi^{\prime} \chi^{\prime}-\frac{\partial V}{\partial \phi} a^{2} \chi+a^{2} \delta p\right)
\end{aligned}
$$




$$
\begin{gathered}
\chi^{\prime \prime}+(d-1) \frac{a^{\prime}}{a} \chi^{\prime}+\left(a^{2} \frac{\partial^{2} V}{\partial \phi^{2}}-\nabla^{2}\right) \chi= \\
=2(d-1) \phi^{\prime} \psi^{\prime}-2(d-2)\left[\frac{c}{2}(\rho-d p)+\frac{\partial V}{\partial \phi}\right] a^{2} \psi-\frac{c}{2} a^{2}(\delta \rho-d \delta p)
\end{gathered}
$$

The linear system formed by the four coupled equations (3.8)-(3.11) determines the classical evolution of four independent perturbation variables $\psi, \chi, \delta \rho$ and $\delta u$ (an additional relation between $\delta p$ and $\delta \rho$ is to be provided by the detailed model of matter sources). In the absence of dilaton background $(\phi=0=\chi)$ one recovers the usual system of equations for hydrodynamical perturbations [30], while in the absence of fluid sources $\left(T_{\mu}^{\nu}=0=\delta T_{\mu}^{\nu}\right)$ one has the usual perturbation system for a scalar field minimally coupled to the metric [30].

When $\phi$ and $T_{\mu \nu}$ are both non-vanishing, and $\delta p$ can be parametrized in terms of $\delta \rho$ as $\delta p=\epsilon(t) \delta \rho$, we may eliminate $\delta \rho$ by means of eq.(3.9), and the system reduces to a pair of second-order differential equations (3.10), (3.11) for the coupled variables $\psi$ and $\chi$. By introducing the bi-dimensional vector

$$
Z=\left(\begin{array}{l}
\psi \\
\chi
\end{array}\right)
$$

and by the dilaton background as

$$
\phi=\beta \ln a, \quad \beta=\text { const }
$$

the abovementioned pair of equations can be represented in compact form as

$$
Z_{k}^{\prime \prime}+2 \frac{a^{\prime}}{a} A Z_{k}^{\prime}+\left(k^{2} B+C\right) Z_{k}=0
$$

where $Z_{k}=\left(\psi_{k}, \chi_{k}\right)$ represents the Fourier component of the perturbation variables, $\nabla^{2} Z_{k}=-k^{2} Z_{k}$, and

$$
\begin{gathered}
A=\left(\begin{array}{cc}
\frac{1}{2}(2 d-3+d \epsilon) & -\frac{1-\epsilon}{4(d-1)} \beta \\
-(d-1)\left[\frac{c d}{2}(1-d \epsilon)+\beta\right] & \frac{1}{2}(d-1)-\frac{c}{4}(1-d \epsilon) \beta
\end{array}\right) \\
B=\left(\begin{array}{cc}
\epsilon & 0 \\
-c(1-d \epsilon)(d-1) & 1
\end{array}\right) \\
C=\left(\begin{array}{cc}
2(d-2) \frac{a^{\prime \prime}}{a}+(d-2)\left[d-4+d \epsilon+\frac{1-\epsilon}{2(d-1)} \beta^{2}\right]\left(\frac{a^{\prime}}{a}\right)^{2} & 0 \\
-c d(d-2)(\gamma-\epsilon)\left[d(d-1)-\frac{\beta^{2}}{2}\right]\left(\frac{a^{\prime}}{a}\right)^{2} & 0
\end{array}\right)
\end{gathered}
$$

(we have neglected here the possible contribution of the dilaton potential, by putting $\left.\partial V / \partial \phi=0=\partial^{2} V / \partial \phi^{2}\right)$. We note that a system of coupled scalar perturbation equations similar to (3.14) was previously considered also in Refs.[31,32] 
where, however, a scalar field model of source ("inflaton" matter) was used, instead of the fluid model adopted in this paper.

Without further approximations, $\psi$ and $\chi$ are thus in general non-trivially mixed, with time-dependent mixing coefficients determined by the explicit model of sources, $\gamma=p / \rho, \epsilon=\delta p / \delta \rho$, and by the background kinematics, $a(t), \phi(t)$, according to eq.(3.14). The solution of (3.14) provides in turn, for any given background configuration, a unique determination of the density contrast $\delta \rho / \rho$ through eq.(3.9), and of the velocity perturbation $\delta u_{i}$ through eq.(3.8).

Eqs.(3.8)-(3.11) are linear in the perturbations and just describe their classical evolution without specifying their absolute magnitude. As clearly stressed in Ref.[30] (see also Refs.[33,34]), in order to determine the absolute magnitude of the vacuum fluctuations and their spectral distribution, one must express the perturbations in terms of the correctly normalized variables satisfying canonical commutation relations. These can be determined by expanding the action to second order in the fluctuations.

For the pure metric-scalar field system $\left(T_{\mu}^{\nu}=0\right)$ such a canonical variable is known to be fixed by the following linear combination of $\chi$ and $\psi$ [35-37]

$$
v=a \chi+z \psi, \quad z=\frac{a^{2} \phi^{\prime}}{a^{\prime}}
$$

For a pure fluid source $(\phi=0)$, with constant $\epsilon$, the canonical variable is instead $[38,39]$

$$
w=\frac{1}{6}(f-\xi \psi), \quad \xi=\frac{a^{3}}{a^{\prime}} \sqrt{\frac{\rho+p}{\epsilon}}
$$

where $f$ is the velocity potential determining the fluid perturbations as

$$
\begin{aligned}
& a \sqrt{\rho+p} \delta u_{i}=-\sqrt{\epsilon} \partial_{i} f \\
& a^{2} \delta \rho=-\frac{a^{2}}{\epsilon}(\rho+p) \psi-\frac{1}{a^{2}}\left(\frac{a^{2} f \sqrt{\rho+p}}{\sqrt{\epsilon}}\right)^{\prime}
\end{aligned}
$$

(we have assumed $d=3$ in the previous three equations). The variables $v, w$ play the role of "normal coordinates" decoupling the system of perturbation equations, and reducing the action to the free scalar field form [30]. Only when $\psi$ is expressed in terms of such variables one gets a canonical normalization of the Fourier modes $\psi_{k}$, and then the correlation function for the metric fluctuations

$$
\left\langle\psi(x) \psi\left(x^{\prime}\right)\right\rangle=\int \frac{d k}{k} \frac{\sin k r}{k r}\left|\delta_{\psi}(k)\right|^{2}
$$


provides the correct spectral distribution for the metric

$$
\left|\delta_{\psi}(k)\right|^{2}=k^{3}\left|\psi_{k}\right|^{2}
$$

and for the dilaton, $\delta_{\chi}(k)$, through eq.(3.16).

If $T_{\mu}^{\nu}$ and $\phi$ are both non-vanishing, one could try a perturbative approach to the spectrum (like in Refs. $[32,40]$ ), by keeping fixed the definitions of $v$ and $w$ as a zeroth-order approximation. In such case, the constraint (3.8) gives (in $d=3$ )

$$
\psi^{\prime}+\frac{a^{\prime}}{a} \psi=\frac{1}{4} \phi^{\prime} \chi-\frac{1}{4} \sqrt{\epsilon(\rho+p)} f
$$

By eliminating $f$ and $\chi$ in terms of $v$ and $w$ through eqs.(3.16), (3.17), by using the constraint (3.21) and the background field equations, one can then express the Fourier mode $\psi_{k}$, from eq.(3.9), as

$$
\psi_{k}=\psi_{k}\left(v, v^{\prime}, w, w^{\prime}, k\right)
$$

Moreover, the system of equations formed by eq.(3.11) and by the combination of eqs.(3.9), (3.10) obtained by eliminating $\delta \rho$, can be written as a system of two second-order differential equations for the coupled modes $v_{k}$ and $w_{k}$. Its solutions, when inserted into eq.(3.22), provide a first approximation to the scalar perturbation spectrum (3.20). From eq.(3.17) one has then the corresponding dilaton spectrum, $\left|\delta_{\chi}\right|=k^{3 / 2}\left|\chi_{k}\right|$, and from eqs.(3.18), (3.17) the density perturbation spectrum $\left|\delta_{\rho}\right|=k^{3 / 2}\left|(\delta \rho / \rho)_{k}\right|$.

In general, dilaton and metric fluctuations will have different spectral distributions, $\left|\delta_{\psi}\right| \neq\left|\delta_{\chi}\right|$. The coupled system of equations is rather complicated, but it seems possible, in principle, to obtain a large variety of spectra as the equation of state and the ratio $\delta p / \delta \rho$ are appropriately varied [41].

In this paper we shall consider a model (see Sec.4 for its motivations) in which the universe evolves from a three-dimensional, dilaton-dominated phase of the pre-big-bang type (with negligible fluid sources, $T_{\mu}^{\nu}=0=\delta T_{\mu}^{\nu}$ ), to the standard radiation-dominated phase $(p=\rho / 3)$, adiabatic $(\epsilon=1 / 3)$, and with frozen Newton constant $(\phi=$ const). More complicated scenarios will be analyzed in future works [41]. The phase of pre-big-bang inflation is assumed to extend in time from $-\infty$ up to the time $\eta=-\eta_{1}<0$, which marks a sudden transition to the phase of radiation dominance. For $\eta<-\eta_{1}$ the constraint (3.8) thus becomes

$$
\psi_{k}^{\prime}+\frac{a^{\prime}}{a} \psi_{k}^{\prime}=\frac{1}{4} \phi^{\prime} \chi_{k}
$$


where, according to eq.(3.16),

$$
\chi_{k}=\frac{v_{k}}{a}-\frac{z}{a} \psi_{k}
$$

When the constraint is inserted into eq.(3.9), and eq.(3.24) is used in order to eliminate $\chi$ and $\chi^{\prime}$, we are led to a relation of the form (3.22), namely

$$
\psi_{k}=-\frac{1}{4 k^{2}} \phi^{\prime} \frac{z}{a}\left(\frac{v_{k}}{z}\right)^{\prime}
$$

In the absence of matter sources, eq.(3.11) becomes equivalent to the combination of eqs.(3.9) and (3.10). By expressing $\chi_{k}$ in terms of $v_{k}$ according to eq.(3.24), and by eliminating $\psi^{\prime}, \psi^{\prime \prime}$ through eq.(3.23), we finally get the canonical perturbation equation [30], valid for $\eta<-\eta_{1}$,

$$
v_{k}^{\prime \prime}+\left(k^{2}-\frac{a^{\prime \prime}}{a}\right) v_{k}=0
$$

In the second, radiation-dominated phase $\left(\eta>-\eta_{1}\right)$, we assume that the dilaton acquires a mass $m$, and it stays frozen at the minimum of the potential (with possible small oscillations around it), so that

$$
V=0=\frac{\partial V}{\partial \phi}, \quad \frac{\partial^{2} V}{\partial \phi^{2}}=m^{2}
$$

In this case $\chi$ decouples from the metric fluctuations (see eqs.(3.8)-(3.11)), that are coupled now to the fluid perturbations only; the canonical variable for their quantization is thus given by eq.(3.17). As $a^{\prime \prime} / a=0$ in the radiation phase, it turns out however that for $\eta>-\eta_{1}$ both $w$ and $a \chi$ satisfy the free oscillator equation, $w^{\prime \prime} / w=$ const (apart from the dilaton mass term, assumed to be negligible at early enough times, see Sec.5). As a consequence, $\psi$ and $\chi$ will have the same spectrum (identical, in this case, to the tensor perturbation spectrum), which can be computed by adopting a second quantization approach, regarding the amplification of the perturbations as a process of particle production from the vacuum, under the action of the cosmological background fields [30].

The Bogoliubov coefficients $c_{ \pm}$for such a process are obtained by matching the solution of eq.(3.25) to a general solution of the plane-wave type,

$$
v_{k}=\frac{1}{\sqrt{k}}\left(c_{+} e^{-i k \eta}+c_{-} e^{i k \eta}\right)
$$

valid for $\eta>-\eta_{1}$. By assuming, for $\eta<-\eta_{1}$, that

$$
a \sim(-\eta)^{-\alpha}, \quad \phi=\beta \ln a
$$


we have

$$
\frac{z^{\prime \prime}}{z}=\frac{a^{\prime \prime}}{a}=\frac{\alpha(\alpha+1)}{\eta^{2}}
$$

The solution of eq.(3.25) describing oscillations with positive frequency at $\eta=-\infty$, and defining the initial vacuum state, is thus given in terms of the second kind Hankel function, $H^{(2)}$, as

$$
v_{k}=\eta^{1 / 2} H_{\nu}^{(2)}(k \eta), \quad \nu=\alpha+\frac{1}{2}
$$

The continuity of $v_{k}$ and $v_{k}^{\prime}$ at the transition time $\eta=-\eta_{1}$ fixes the Bogoliubov coefficient $c_{-}(k)$, and the corresponding expectation number of particles produced in the mode $k$. For $k \lesssim 1$ we obtain

$$
\langle n(k)\rangle=\left|c_{-}(k)\right|^{2} \simeq\left(k \eta_{1}\right)^{-2|\nu|-1}
$$

(higher mode production turns out to be exponentially suppressed [3, 42], and can be neglected for the purpose of this paper). In terms of the proper frequency $\omega=k / a$, the energy density $\rho_{\chi}$ of the produced dilatons is thus characterized by a spectral distribution $\omega d \rho_{\chi} / d \omega \simeq \omega^{4}\left|c_{-}\right|^{2}$ which may be written, in units of critical density $\rho_{c}=H^{2} / G$,

$$
\Omega_{\chi}(\omega, t)=\frac{\omega}{\rho_{c}} \frac{d \rho_{\chi}}{d \omega} \simeq \frac{G \omega^{4}}{H^{2}}\left(\frac{\omega}{\omega_{1}}\right)^{-2-2 \alpha}=G H_{1}^{2}\left(\frac{\omega}{\omega_{1}}\right)^{2-2 \alpha}\left(\frac{H_{1}}{H}\right)^{2}\left(\frac{a_{1}}{a}\right)^{4}
$$

where $\omega_{1}(t)=H_{1} a_{1} / a(t)$ is the proper frequency of the highest excited mode (here we have supposed $\alpha \geq-1 / 2)$. This is the same spectrum as that obtained in the graviton case [43], with an intensity normalized to the final inflation scale $H_{1}$. It is growing for a phase of superinflationary pre-big-bang expansion $(\alpha<1)$, flat for de Sitter $(\alpha=1)$, and decreasing for power-law inflation $(\alpha>1)$.

It should be stressed that this second quantization approach is convenient to discuss the squeezing properties of the produced radiation [3,34,44-47] but, for what concerns the perturbation spectrum, it is completely equivalent to the more traditional approach in which one computes the parametric amplification of the perturbation amplitude. In this second approach one has indeed, according to the "effective potential" $z^{\prime \prime} / z$ of eq.(3.25), a mode amplitude which is constant, $\left|v_{k}\right| \simeq 1 / \sqrt{k}$, in the initial region $\eta \rightarrow-\infty$ where $k^{2}>>\left|z^{\prime \prime} / z\right| \simeq \eta^{-2}$, and which grows with power-like behaviour in $\eta$ in the non-oscillatory region defined by $k^{2}<<\left|z^{\prime \prime} / z\right|$ (in the subsequent radiation era the solution for $v$ is again oscillating, with frozen amplitude). In the non-oscillatory region

$$
v_{k}=c_{1} z+c_{2} z \int^{\eta} \frac{d \eta^{\prime}}{z^{2}\left(\eta^{\prime}\right)}-k^{2} z \int^{\eta} \frac{d \eta^{\prime}}{z^{2}\left(\eta^{\prime}\right)} \int^{\eta^{\prime}} d \eta^{\prime \prime} z^{2}\left(\eta^{\prime \prime}\right)+O\left(k^{4}\right)
$$


$\left(c_{1}, c_{2}\right.$ are integration constants) is the general solution of eq.(3.25) to first order in $k^{2}$ (the first sub-leading term has been included to have non-trivial derivative of $v / z$ ). This gives, for the background (3.28) (with obvious redefinition of $c_{1}, c_{2}$, and introducing a further numerical constant $\left.c_{3}\right)$,

$$
v_{k}=c_{1}|\eta|^{-\alpha}+c_{2}|\eta|^{1+\alpha}-c_{3} k^{2}|\eta|^{2-\alpha}
$$

For $\alpha>0$ (inflationary expansion) the first term is the dominant one in the the $|\eta| \rightarrow 0$ limit, and the wave amplification achieved in this limit can thus be estimated as [30]

$$
v_{k} \simeq \frac{v_{k}(\eta)}{\sqrt{k}}\left(\frac{1}{v_{k}}\right)_{k \simeq a H} \simeq \frac{z}{\sqrt{k}}\left(\frac{1}{v_{k}}\right)_{k \simeq a H}\left[1-\frac{k^{2} \eta^{2}}{2(1-2 \alpha)}\right]
$$

The variable at the denominator is to be evaluated at the time $\eta \simeq k^{-1}$, where the mode $k$ "hits" the effective potential barrier $z^{\prime \prime} / z \simeq \eta^{-2}$ (otherwise stated: at the time of first horizon crossing, when $H=\omega)$. By inserting this value of $v$ into eqs.(3.25) and (3.20), and recalling the definition of $z$, we are led to

$$
\left|\delta_{\psi}(k)\right|^{2} \simeq k^{3}\left|\psi_{k}\right|^{2} \simeq\left(\frac{k}{z}\right)_{k \simeq a H}^{2} \simeq\left(\frac{H^{2}}{\dot{\phi}}\right)_{k \simeq a H}^{2}
$$

which is the standard expression for the scalar perturbation spectrum $[35,48]$ associated with the inflation-radiation transition (see Sec. 4 for a proof of the fact that the same result is recovered in the case of contracting backgrounds). The same spectrum is obtained for the dilaton perturbations as we have, from eq.(3.24),

$$
\left|\delta_{\chi}\right|^{2}=k^{3}\left|\frac{v_{k}}{a}-\frac{z}{z} \psi_{k}\right|^{2} \simeq\left(\frac{k}{z}\right)_{k \simeq a H}^{2} \simeq\left|\delta_{\psi}\right|^{2}
$$

It is important to stress that this expression, when multiplied by $G$, exactly coincides with the spectral energy density (3.32) (modulo numerical factors of order unity), evaluated in the radiation era. Indeed, multiplying and dividing eq.(3.37) by $H_{1}^{2} \simeq\left(a_{1} \eta_{1}\right)^{-2}$ we have

$$
\begin{gathered}
G\left|\delta_{\chi}\right|^{2} \simeq G\left(\frac{H^{2}}{\dot{\phi}}\right)_{k \simeq a H}^{2} \simeq\left(\frac{G}{a \eta}\right)_{k \simeq a H}^{2} \simeq G H_{1}^{2}\left(\frac{a_{1} \eta_{1}}{a \eta}\right)_{k \simeq a H}^{2} \\
\simeq G H_{1}^{2}\left(k \eta_{1}\right)^{2-2 \alpha}=G H_{1}^{2}\left(\frac{\omega}{\omega_{1}}\right)^{2-2 \alpha} \simeq\left(\frac{\omega}{\rho_{c}} \frac{d \rho_{\chi}}{d \omega}\right)_{\mathrm{rad}}
\end{gathered}
$$

in agreement with eq.(3.32) for $a \sim t^{1 / 2} \sim H^{-1 / 2}$. 
As already stressed in Ref.[14] for the tensor perturbation case, we want to remark finally that the scalar perturbation spectrum is the same in the E and BD frame, as a consequence of the equality of the two conformal time coordinates (see Sec.2). Indeed, quite independently of the computational method (first or second quantization) the spectral behaviour of the energy density is fixed by the Bessel index $\nu$ of the solution of eq.(3.25), which depends, in turn, on the slope of the effective potential $z^{\prime \prime} / z$. For a generic $d=3$ background in the $\mathrm{E}$ frame we have (recall eq.(2.55))

$$
\alpha(\gamma)=\frac{\gamma-1}{1-2 \gamma+3 \gamma^{2}}
$$

so that, according to eq.(3.29)

$$
\left(\frac{z^{\prime \prime}}{z}\right)_{E}=\left(\frac{a^{\prime \prime}}{a}\right)_{E}=\frac{\gamma}{\eta_{E}^{2}} \frac{(\gamma-1)(3 \gamma-1)}{\left(1-2 \gamma+3 \gamma^{2}\right)^{2}}
$$

Eq.(3.25) for $v_{k}$ is not conformally invariant, and in the BD frame the effective potential becomes

$$
\left(\frac{z^{\prime \prime}}{z}\right)_{B D}=\left(\frac{z^{\prime \prime}}{z}\right)_{E}\left(a_{B D}, \phi_{B D}\right)=\left(\frac{a^{\prime \prime}}{a}\right)_{B D}-\phi_{B D}^{\prime}\left(\frac{a^{\prime}}{a}\right)_{B D}-\frac{1}{2} \phi_{B D}^{\prime \prime}+\frac{1}{4} \phi_{B D}^{\prime 2}
$$

In this frame, however, the spectrum is determined by the conformally transformed backgrounds, namely by the solutions $(2.46),(2.47)$ of the BD field equations. By inserting their explicit expressions for $d=3$ we get

$$
\left(\frac{z^{\prime \prime}}{z}\right)_{B D}=\frac{\gamma}{\eta_{B D}^{2}} \frac{(\gamma-1)(3 \gamma-1)}{\left(1-2 \gamma+3 \gamma^{2}\right)^{2}}
$$

which coincides with the effective E-frame potential (3.40) because of the equality $\eta_{B D}=\eta_{E}$. The same results holds for a dilaton-driven evolution, described by the solution (2.57) and their BD-transformed expressions.

\section{Pre-big-bang scenario in the Brans-Dicke and Einstein frame.}

As seen in the previous Section, the spectral distribution of the perturbations is uniquely fixed by the explicit form of the background solution. The timeevolution of the background fields is determined, in turn, by the particular model of matter sources. As in our previous work [20,27], our model of sources consists of 
a sufficiently diluted gas of classical fundamental strings whose mutual interactions are described, in a mean-field approximation sense, as the interaction of each single string with the background generated by all the others according to the tree-level effective action (2.4). The source stress-tensor appearing in eq.(2.1) is thus given by a sum over all strings (labelled by $i$ ) of the stress-tensor of each individual string $T_{i}^{\mu \nu}$, where

$$
T_{i}^{\mu \nu}(x)=\frac{1}{\pi \alpha^{\prime} \sqrt{|g|}} \int d \sigma d \tau\left(\frac{d X_{i}^{\mu}}{d \tau} \frac{d X_{i}^{\nu}}{d \tau}-\frac{d X_{i}^{\mu}}{d \sigma} \frac{d X_{i}^{\nu}}{d \sigma}\right) \delta^{D}(X-x)
$$

and, for each $i$, the coordinates $X^{\mu}$ satisfy the string equations of motion in the given background,

$$
\begin{gathered}
\frac{d^{2} X^{\mu}}{d \tau^{2}}-\frac{d^{2} X^{\mu}}{d \sigma^{2}}+\Gamma_{\alpha \beta}^{\mu}\left(\frac{d X^{\alpha}}{d \tau}+\frac{d X^{\alpha}}{d \sigma}\right)\left(\frac{d X^{\beta}}{d \tau}-\frac{d X^{\beta}}{d \sigma}\right)=0 \\
g_{\mu \nu}\left(\frac{d X^{\mu}}{d \tau} \frac{d X^{\nu}}{d \tau}+\frac{d X^{\mu}}{d \sigma} \frac{d X^{\nu}}{d \sigma}\right)=0, \quad g_{\mu \nu} \frac{d X^{\mu}}{d \tau} \frac{d X^{\nu}}{d \sigma}=0
\end{gathered}
$$

Here $\left(2 \pi \alpha^{\prime}\right)^{-1}$ is the string tension, $\Gamma_{\mu \nu}^{\alpha}$ the Christoffel symbol for the background metric $g_{\mu \nu}, \tau$ and $\sigma$ the usual world-sheet time and space variables (we are using the gauge in which the world-sheet metric is conformally flat).

The general exact solution of the system of equations (2.1)-(2.3), (4.1), (4.2) is hard to find and certainly impossible to express in closed form. In some appropriate asymptotic regime, however, the solution of the string equations of motion, when inserted into the energy-momentum tensor (4.1), provides an effective equation of state which allows to describe the string sources in the perfect fluid approximation [20,27], and to recover then the general background solutions of Sec.2. The cosmological solution we are looking for is characterized in particular by having, as initial configuration, the string perturbative vacuum, namely flat spacetime with vanishing torsion and coupling constant, $H_{\mu \nu \alpha}=0, \phi=-\infty$. In this regime strings move freely, do not decay and behave as a pressureless gas with an energy density $\rho$. We shall assume $\rho$ to be small enough initially so that, as we shall see, it will represent a negligible source of curvature. On the other hand a finite $\rho$ is certainly sufficient to make the dilaton evolve away from the perturbative minimum.

Indeed, the negative branch $\left(x \leq x_{-}\right)$of the general background solution with perfect fluid sources, eqs.(2.37)-(2.31), may be written in the case of vanishing pressure $\left(\gamma_{i}=0\right)$ as

$$
a_{i}(t)=a_{i 0}\left|\frac{t-2 T}{t}\right|^{\alpha_{i}}, \quad e^{\bar{\phi}}=\frac{16 L^{2} e^{-\phi_{0}}}{\mid(t(t-2 T) \mid}, \quad \bar{\rho}=\frac{1}{L} \frac{d x}{d t}=\frac{e^{\phi_{0}}}{4 L^{2}}=\text { const }
$$




$$
\alpha_{i}=\frac{t_{i}}{T}, \quad T=\left(\sum_{i} t_{i}^{2}\right)^{1 / 2}, \quad t \leq 0
$$

$\left(t_{i}\right.$ are integration constants, and we have performed a time translation to shift the singularity from $x=x_{-}$to the origin, by choosing $\left.x_{0}=-T\left(e^{\phi_{0}} / 4 L\right)\right)$. This background is certainly consistent with the solution of the string equations of motion (4.2) in the $t \rightarrow-\infty$ limit. Indeed, in this limit, the metric is flat,

$$
a_{i}=\text { const }, \quad \phi \sim-2 \ln (-t), \quad \rho=\text { const }
$$

and the solutions of eqs.(4.2) are characterized by $\sum_{i}\left(d x^{i} / d \tau\right)^{2}=\sum_{i}\left(d x^{i} / d \sigma\right)^{2}$. Eq.(4.1) gives then $T_{0}^{0}=$ const, $T_{i}^{i}=0$, namely a stress tensor describing dust-like matter in the perfect fluid approximation. For $t \sim-T$, however, the curvature scale begins to increase, the string sources progressively enter a non-oscillating unstable regime [27], and one must then take into account the fact that the ratios $\gamma_{i}=p_{i} / \rho$ begin to evolve in time.

In connection with this last point we note that the solution (4.3), which, for $t \leq 0$, gives

$$
H_{i}=\frac{2 t_{i}}{t(t-2 T)}, \quad \dot{\bar{\phi}}=-\frac{2(t-T)}{t(t-2 T)}, \quad \bar{\rho} e^{\bar{\phi}}=\frac{4}{t(t-2 T)}=\rho e^{\phi},
$$

is characterized by two scales. One is the curvature scale $\left(\left|H_{T}\right| \sim T^{-1}\right.$ at $\left.t \sim-T\right)$ at which the transition from flat to curved spacetime regime occurs and inflation begins. At $t \sim-T$, the curvature $H^{2}$ is of the same order as $\dot{\bar{\phi}}^{2}$ or $\rho e^{\phi}$ while, much earlier, it was negligible. By contrast, much later than $t=-T$, it is $\rho e^{\phi}$ which becomes negligible and one recovers the vacuum solutions. $T$ is a free phenomenological parameter of the solution. The other scale is the maximal scale $\left|H_{1}\right| \sim t_{1}^{-1}$, at the time $t \sim-t_{1}$, after which the solution is no longer valid, because higher orders in $\alpha^{\prime}$ have to be added to the low energy effective action (2.4). This final scale $t_{1}$ is thus determined by the string tension as $t_{1} \simeq \sqrt{\alpha^{\prime}}=\lambda_{s}$, where $\lambda_{s}$ is the fundamental (minimal) length parameter of string theory [49], which may be assumed to coincide roughly with the present value of the Planck length, $l_{p}=M_{p}^{-1}$. The important point to stress is that, in any realistic inflationary scenario, $T$ and $\lambda_{s}$ cannot be of the same order as we will now show.

When $|t|<T$, the solution describes an accelerated evolution given asymptotically by

$$
a_{i}(t) \sim(-t)^{-\alpha_{i}}, \quad\left|\alpha_{i}\right|<1, \quad \sum_{i} \alpha_{i}^{2}=1
$$


and which is of the type given in eq.(2.42) (we call "accelerated" a configuration in which $\dot{a}, \ddot{a}$ and $\dot{H}$ have the same sign, positive for expansion, negative for contraction $[2,23,27])$. In this metric, the particle horizon along any given spatial direction,

$$
d_{p}^{i}(t)=a_{i}(t) \int_{-\infty}^{t} d t^{\prime} a_{i}^{-1}\left(t^{\prime}\right)
$$

evolves for $|t|<<T$ like the scale factor, $d_{p}^{i} \sim a_{i}$, while the event horizon

$$
d_{e}^{i}(t)=a_{i}(t) \int_{t}^{0} d t^{\prime} a_{i}^{-1}\left(t^{\prime}\right)
$$

shrinks linearly in time, $d_{e}^{i} \sim(-t)$, for $t \rightarrow 0$. The ratio of the two proper sizes $r^{i}(t)=d_{p}^{i} / d_{e}^{i}$ thus grows in time, for $|t|<<T$, as $(-t)^{-\alpha_{i}-1} \sim(-\eta)^{-1}$. On the other hand, the horizon problem of the standard cosmological model [50] is solved if, for every spatial direction, the growth of the ratio $r^{i}(t)$ when $|t|$ is ranging from $T$ to $t_{1}$, is large enough to compensate the decreasing of the ratio in the subsequent decelerated phase down to the present time $t_{0}$. This implies, in the hypothesis that the pre-big-bang era is followed by the standard radiation-dominated (until $t=t_{2}$ ) and matter-dominated evolution,

$$
\begin{gathered}
\left(\frac{t_{1}}{T}\right)^{-\alpha_{i}-1} \simeq\left(\frac{H_{T}}{H_{1}}\right)^{-\alpha_{i}-1} \gtrsim\left(\frac{a_{1}}{a_{0}}\right)\left(\frac{t_{0}}{t_{1}}\right)= \\
=\left(\frac{t_{1}}{t_{2}}\right)^{-1 / 2}\left(\frac{t_{2}}{t_{0}}\right)^{-1 / 3}=\left(\frac{H_{1}}{H_{2}}\right)^{1 / 2}\left(\frac{H_{2}}{H_{0}}\right)^{1 / 3} \simeq 10^{30} \sqrt{\frac{H_{1}}{M_{p}}}
\end{gathered}
$$

(the same condition is required to solve the flatness problem, see below). For an expanding $d$-dimensional isotropic background $\alpha_{i}=1 / \sqrt{d}$ (see eq.(2.48)), and the previous condition gives in particular, for $t_{1} \simeq \lambda_{s} \simeq M_{p}^{-1}$,

$$
T \gtrsim 10^{30 \sqrt{d} /(\sqrt{d}+1)} \lambda_{s}, \quad H_{T} \lesssim 10^{-30 \sqrt{d} /(\sqrt{d}+1)} M_{p}
$$

We shall thus assume that the scale $T$ appearing in the solution (4.3) is much larger than the string scale $\lambda_{s} \simeq M_{p}^{-1}$.

This fact has an important consequence. In this case the background (4.3) becomes in fact a good zeroth-order approximation to the general solution of the full system of equations, consistent with the string equations of motion not only in the asymptotic limit $t \rightarrow-\infty$.

By adopting an iterative approach, let us assume indeed the solution (4.3) to be a zeroth-order approximation, and let us compute the first order corrections by 
inserting that solution into the string equations of motion, in order to obtain the corresponding value of $\gamma_{i}(t)$. To this aim we observe that the given background is characterized, asymptotically, by an accelerated metric with shrinking event horizons (see eq.(4.6)). We recall that, in such a background, the string equations of motion admit oscillating solutions, corresponding to strings with constant proper size $L_{s}$, provided $L_{s}$ is smaller than the size of the event horizon $\sim H^{-1}(t)$ (stable strings), while the solutions describe non oscillating strings with $L_{s}(t) \sim a(t)$ if $L_{s}>H^{-1}$ (unstable strings) [27].

The evolution of a network of strings with some initial distribution in backgrounds of the type discussed above can be investigated [51]. One can show that the number $n\left(L_{s}, t\right)$ of strings (per unit length) of given size $L_{s}$, at time $t$, must satisfy in the given background the approximate evolution equation [51]

$$
\frac{\partial n}{\partial t}=-H \frac{\partial}{\partial L_{s}}\left[n L_{s} \theta\left(L_{s}-H^{-1}\right)\right]
$$

where $\theta$ is the Heaviside step function. Its general solution can be written in implicit form as [51]

$$
\begin{gathered}
n\left(L_{s}, a(H)\right)=n_{0}\left(L_{s}\right) \theta\left(H^{-1}-L_{s}\right)+f\left(\frac{L_{s}}{a}\right) \theta\left(L_{s}-H^{-1}\right), \\
f\left(\frac{H^{-1}}{a}\right)=n_{0}\left(H^{-1}\right)\left(1+\frac{\partial \ln a}{\partial \ln H}\right)^{-1}
\end{gathered}
$$

where $n_{0}$ is the initial string distribution.

The energy associated at a time $t$ with stable $\left(\bar{\rho}_{S}\right)$ and unstable $\left(\bar{\rho}_{U}\right)$ strings can be estimated as

$$
\bar{\rho}_{S} \sim \int_{\lambda_{s}}^{H^{-1}} L_{s} n\left(L_{s}, t\right) d L_{s}, \quad \bar{\rho}_{U} \sim \int_{H^{-1}}^{\infty} L_{s} n\left(L_{s}, t\right) d L_{s}
$$

However, for a perfect gas of stable strings $p_{S}=0$, while, for unstable strings, $p_{U}=$ $\pm \rho_{U} / d$, with the sign fixed by the exponent $\alpha_{i}$ of eq.(4.6), $\operatorname{sign}\left\{p_{U}\right\}=-\operatorname{sign}\left\{\alpha_{i}\right\}$, as discussed in [27]. Therefore, the ratio $\gamma=p / \rho$ as a function of time, for a perfect gas of strings in an accelerated metric background, can be approximated as

$$
\gamma(t)= \pm \frac{1}{d} \frac{\bar{\rho}_{U}}{\bar{\rho}_{U}+\bar{\rho}_{S}}
$$

By inserting into eqs.(4.13), (4.14) the solution (4.12) expressed for our particular metric (4.6), with an initial string distribution $n_{0}\left(L_{s}\right) \sim L_{s}^{-3}$, one then finds for each spatial direction [51]

$$
\gamma_{i}(t)=-\frac{1}{d} \lambda_{s} H_{i}(t)
$$


where $H_{i}$ is given by eq.(4.5). The above result is valid for $\left|H_{i}\right|<\lambda_{s}^{-1} \simeq M_{p}$ and is not very sensitive to the initial string distribution.

We insert now this expression into the right-hand side of the field equations (2.18), (2.19), by recalling that, for the pressureless background (4.3) one has, to zeroth-order,

$$
\Gamma_{i}^{(0)}=x_{i}=\frac{e^{\phi_{0}}}{4 L} t_{i}
$$

Then, to next order,

$$
\begin{gathered}
\Gamma_{i}=x_{i}+\int_{-\infty}^{x} \gamma_{i}\left(x^{\prime}\right) d x^{\prime}=\frac{e^{\phi_{0}}}{4 L}\left[t_{i}-\frac{\lambda_{s}}{d} \int_{-\infty}^{t} \frac{d a_{i}}{a_{i}}\right]= \\
=\Gamma_{i}^{(0)}\left[1-\frac{\lambda_{s}}{d T} \ln \left(\frac{t-2 T}{t}\right)\right]
\end{gathered}
$$

According to our iterative approach, the integration of eqs.(2.18), (2.19) with $D(x)$ determined by this new expression for $\Gamma_{i}$ provides a first order approximation to the background fields $a(t), \phi(t)$. The corrections to the solution (4.3) due to a nonvanishing effective pressure of the string gas are certainly negligible for $|t|>>T$, in the regime in which the background (4.3) satisfies $H^{2}<<\rho e^{\phi} \sim \dot{\bar{\phi}}^{2}$. However, as clearly shown by eq.(4.17), if $T>>\lambda_{s}$ then the first order corrections keep small also in the $t \sim-T$ regime, in which $H^{2} \sim \rho e^{\phi} \sim \dot{\bar{\phi}}^{2}$, and even in the limit $t \rightarrow-t_{1} \simeq \lambda_{s}$, in which $\rho e^{\phi}<<\dot{\bar{\phi}}^{2} \sim H^{2}$. Within the assumption that $T$ is very large in string units, the solution (4.3) becomes then a good approximation to the exact solution of the system of background equations and string equations of motion, for the whole range $-\infty \leq t \leq-t_{1} \simeq \lambda_{s}$.

We stress that, in this scenario, when $|t|<<T$ the source term $\rho e^{\phi}$ becomes negligible with respect to $H^{2}$ and $\dot{\bar{\phi}}^{2}$ (see eq.(4.5)); quite independently of the exact value of the pressure and of the particular type of equation of state at the scale $T$, the background rapidly converges, for $|t|<<T$, to a phase of vacuum, dilatondriven accelerated evolution (as discussed in Sec.2), described by the metric (4.6). We are left, therefore, with two phenomenological possibilities.

The first is the case in which $T$, and then the temporal extension of the regime (4.6), is much larger than the minimal value fixed by eq.(4.9) to secure a phenomenologically sufficient amount of inflation. This means, in conformal time,

$$
\left|\eta_{T}\right|>>10^{30}\left(\frac{H_{1}}{M_{p}}\right)^{1 / 2}\left|\eta_{1}\right| \simeq\left|\eta_{0}\right|
$$


where $\eta_{0}$ is the time when the largest scale, corresponding to the minimum frequency mode $\omega_{0}=H_{0}$, was pushed out of the event horizon during the pre-bigbang phase. In this case, all today's observable scales crossed the horizon in the dilaton-driven regime (4.6), so that the presently observed perturbation spectrum is wholly determined by the metric behavior of that regime, quite independently of possible earlier matter corrections to the background.

The second possibility is the case of nearly "minimal" inflation, corresponding to the equality in the condition (4.9), which implies then $\left|\eta_{T}\right| \sim\left|\eta_{0}\right|$. In this case the largest scales crossed the horizon when the contribution of the string sources to the metric background was of the same order as the dilaton contribution. As a consequence, the low frequency part of the scalar perturbation spectrum may be affected by the matter corrections, and may be sensitive to the particular type of equation of state. The spectrum is thus to be computed by including the nontrivial mixing induced by the source terms $T_{\mu \nu}$ and their perturbations, $\delta T_{\mu \nu}$, as discussed in Sec.3.

As anticipated in Sec.3, in this paper we will discuss the first possibility only. We shall assume, in particular, that the phase of accelerated evolution responsible for the solution of the standard kinematic problems, and for the amplification of the perturbations (at all presently accessible scales), is described by a threedimensional, isotropic, dilaton-dominated background with

$$
\begin{gathered}
a(t) \sim(-t)^{-1 / \sqrt{3}}, \quad a(\eta) \sim(-\eta)^{-1 /(\sqrt{3}+1)}, \quad \phi \sim(3+\sqrt{3}) \ln a, \\
t \leq-t_{1}<0 \quad \eta \leq-\eta_{1}<0
\end{gathered}
$$

(according to eqs.(2.48), (2.49)). More complicated scenarios, in particular with higher-dimensional, anisotropic, sourceless backgrounds will be analyzed elsewhere [41]).

The metric (4.19) describes superinflationary expansion [52]. In order to obtain the dilaton spectrum, by applying eq.(3.32), we must transform however the solution (4.19) into the E frame, where it takes the form (see eqs.(2.57), (2.58))

$$
a_{E}\left(t_{E}\right) \sim\left(-t_{E}\right)^{1 / 3}, \quad a_{E}(\eta) \sim(-\eta)^{1 / 2}, \quad \phi_{E} \sim-\sqrt{12} \ln a_{E}
$$

This metric describes, for $t \rightarrow 0_{-}$, a contracting background. Potentially, this represents a difficulty of the whole scenario: indeed, the approximation of a diluted string gas might be no longer valid in a contracting background, as well as the approximated expression (3.36) for the perturbation spectrum, obtained in the 
case of inflationary expansion. Most important, it might seem impossible, in a contracting background, to achieve a solution of the standard kinematic problems [50], thus rendering "frame-dependent" the inflationary virtues of the pre-big-bang scenario.

Surprisingly enough, however, this is not the case, as a consequence of the fact that the contraction of the metric (4.20) is of the accelerated type, with $\dot{a}<0$, $\ddot{a}<0, \dot{H}<0$ (one can show, in general, that all the BD solutions describing superinflationary expansion, with or without matter sources, are transformed through the Weyl rescaling (2.52) into E backgrounds whose metric describes accelerated contraction [23]). Let us show, first of all, that a phase of accelerated contraction is equally good to solve the kinematic problems of the standard model as a phase of superinflationary expansion, characterized by $\dot{a}>0, \ddot{a}>0, \dot{H}>0$. Consider indeed the so-called flatness problem: the spatial curvature term becomes negligible with respect to the other terms of the cosmological equations if the ratio

$$
r_{1}(t)=\frac{k}{a^{2} H^{2}}=\frac{k}{\dot{a}^{2}}
$$

goes to zero during inflation. Such a condition is certainly satisfied by a metric which behaves, asymptotically, as

$$
a(t) \sim(-t)^{\alpha}, \quad t<0, \quad \alpha<1
$$

for $t \rightarrow 0_{-}$. For $\alpha<0$ this metric parametrizes the known case of pole-inflation (superinflationary expansion [52]). For $0<\alpha<1$ one has instead accelerated contraction. In both cases the curvature scale is growing, and $H, \dot{H}$ diverge as $t \rightarrow 0_{-}$.

Accelerated contraction can also provide a solution to the horizon problem. Indeed, by recalling the previous definition of particle (eq.(4.7)) and event (eq.(4.8)) horizon, one finds that the ratio of their proper sizes in the background $(4.22)$,

$$
r_{2}(t)=\frac{d_{p}(t)}{d_{e}(t)} \sim(-t)^{\alpha-1},
$$

diverges for $t \rightarrow 0_{-}$. This means that causally connected regions will always cross the horizon, asymptotically, not only in the case of superinflationary expansion $(\alpha<0)$, but also in the case of accelerated contraction $(0<\alpha<1)$.

It is important to stress that the condition for a successful resolution of the horizon and flatness problems, when expressed in conformal time, is exactly the same for superinflation and accelerated contraction. Quite independently of $\alpha$, in 
fact, the ratio $r_{2}$ scales as $\eta^{-1}$, while $r_{1}$ scales as $\eta^{2}$. The horizon problem is solved if $r_{2}$, evaluated at the end of inflation $\left(\eta=\eta_{f}\right)$, is larger than a present value of $r_{2}$ of order unity, rescaled up to $\eta_{f}$. Namely

$$
\frac{\left|\eta_{i}\right|}{\left|\eta_{f}\right|} \gtrsim \frac{\left|\eta_{0}\right|}{\left|\eta_{f}\right|}
$$

where $\eta_{i}$ denotes the beginning of the (contracting or expanding) accelerated evolution (see also eq.(4.9)). The flatness problem is solved if $r_{1}$, at $\eta=\eta_{f}$, is tuned to a small enough value, so that the subsequent decelerated evolution leads to a present value of the ratio $r_{1}\left(\eta_{0}\right) \lesssim 1$. This implies

$$
\left(\frac{\eta_{f}}{\eta_{i}}\right)^{2} \lesssim\left(\frac{\eta_{f}}{\eta_{0}}\right)^{2}
$$

which is clearly equivalent to the previous condition, as expected.

Therefore, if the accelerated phase of pre-big-bang is long enough to solve the kinematic problems in the $\mathrm{BD}$ frame, where the metric describes superinflationary expansion, then the solution holds also in the E frame where the metric describes accelerated contraction, because the conditions are the same in conformal time, and the conformal time is the same in the two frames [23]. (We note, incidentally, that the kinematic problems can thus be solved also if one chooses negative integration constants, $t_{i}<0, \alpha_{i}<0$, for the background solution (4.6), corresponding to a metric describing accelerated contraction already in the BD frame). For the solution of the entropy problem, of course, a non-adiabatic phase associated to the inflation-radiation transition is necessarily required, in addition to the accelerated kinematic, as recently stressed also in [53].

For what concerns the dilution of the string gas, we recall that in the BD frame a model of source as a weakly interacting string network is a very good approximation. In that frame, indeed, by starting at some initial time $t_{i}$ with a packing factor $=$ (average distance/average size) of order unity, one ends up, at any subsequent time $t_{f}$, with a number of strings per unit of string volume which is diluted as $n_{f} / n_{i}=\left(a_{i} / a_{f}\right)^{d}$ ( $<1$ since the metric is expanding). In the E frame the metric is contracting, but the string proper size $L_{s}^{E}(t)$ shrinks with time as $L_{s}^{E}(t)=\left(a_{E} / a\right) \lambda_{s}$, where $a$ is the BD scale factor. As a consequence, the number of strings per unit of string volume scales as $n(t)=\left(L_{s}^{E} / \lambda_{s}\right)^{d} a_{E}^{-d}=a^{-d}$, and it is again diluted as time goes up, exactly by the same amount as in the BD frame. In other words, one finds that, at the end of inflation, a region of space of initial linear dimensions $O\left(\lambda_{s}\right)$ has become exponentially large in Planck units irrespectively of 
the frame one is using. With similar arguments one can show [23] that the heating up of the string gas with respect to the radiation, which is easy to understand in the $\mathrm{BD}$ frame where the metric is expanding and the radiation is red-shifted, also occurs in the $\mathrm{E}$ frame in spite of the fact that the radiation is blue-shifted because of the contraction.

We want to show, finally, that the result (3.36) for the scalar perturbation spectrum is also valid if the transformed metric, in the E frame, is a contracting one. Consider in fact eqs.(3.33), (3.34), for the mode $v_{k}$ in the non-oscillating regime. Since the variable $z=a \dot{\phi} / H$ goes like $a$ for $\phi \sim \beta \ln a$, it might seem that for fast enough contraction the second term of the expansion could dominate the first one, asymptotically, thus changing the perturbation spectrum. We must recall, however, that in the scenario that we are considering the universe evolves from an initial phase of pre-big-bang to the standard, decelerated, radiation-dominated expansion. In the $\mathrm{BD}$ frame the universe is always expanding, $H_{B D}>0$, while in the $\mathrm{E}$ frame an initial contraction $\left(H_{E}<0\right)$ turns into a final expansion $\left(H_{E}>0\right)$, with a necessary turning point of $H_{E}$ at some time $\eta^{\star}$ near the transition time $-\eta_{1}$. On the other hand, the conformal transformation (2.52) gives (in $d=3$ ) $H_{E}=\left(H_{B D}-\dot{\phi} / 2\right) e^{\phi / 2}$ : it follows that $\dot{\phi} \neq 0$ where $H_{E}=0$, and that $z \rightarrow \infty$ for $\eta \rightarrow \eta^{\star}$, so that the first term of the expansion (3.33) is still the dominant one even in the E frame.

By putting, in this frame, $a^{\prime} / a \simeq\left(a^{\prime} / a\right)_{\star}^{\prime}\left(\eta-\eta^{\star}\right)$ for $\eta \rightarrow \eta^{\star}(z \rightarrow \infty)$, the amplification of $\psi_{k}$ in this limit can thus be estimated as (using eq.(3.25))

$$
\left|\psi_{k}\right| \simeq\left|\frac{\phi^{\prime} z}{k^{2} a}\left(\frac{v_{k}}{z}\right)^{\prime}\right| \simeq\left|\frac{\left(\phi_{\star}^{\prime}\right)^{2}}{\sqrt{k}\left(a^{\prime} / a\right)_{\star}^{\prime}}\left(\frac{1}{z}\right)_{k \simeq a H}\right| \simeq \frac{1}{\sqrt{k}}\left(\frac{1}{z}\right)_{k \simeq a H}
$$

where $\phi_{\star}^{\prime} \equiv \phi^{\prime}\left(\eta_{\star}\right)$, and we have used the background equation $\phi^{\prime 2}=-6\left(a^{\prime} / a\right)^{\prime}$. We thus recover for $\left|\delta_{\psi}\right|^{2}=k^{3}\left|\psi_{k}\right|^{2}$ in the E frame the standard result (3.36), in spite of the contracting character of the transformed pre-big-bang metric.

\section{Phenomenological constraints on the dilaton spectrum.}

In the simplified model of pre-big-bang motivated and discussed in the previous Section, and described by the background (4.20), the dilaton perturbations are amplified with a growing spectral distribution: one has, from eq.(3.32),

$$
\Omega_{\chi}(\omega, t) \simeq G H_{1}^{2}\left(\frac{\omega}{\omega_{1}}\right)^{3}\left(\frac{H_{1}}{H}\right)^{2}\left(\frac{a_{1}}{a}\right)^{4}
$$


The total dilaton energy density $\rho_{\chi}(t)$ is thus dominated by the highest frequency mode $\omega_{1}$,

$$
\Omega_{\chi}(t)=\frac{\rho_{\chi}(t)}{\rho_{c}(t)}=\int^{\omega_{1}} \frac{d \omega}{\omega} \Omega_{\chi}(\omega, t) \simeq G H_{1}^{2}\left(\frac{H_{1}}{H}\right)^{2}\left(\frac{a_{1}}{a}\right)^{4}
$$

and since $\rho_{\chi}(t)$ decreases in time like the radiation density $\left(\sim a^{-4}\right)$, its value in critical units, $\Omega_{\chi}(t)$, remains constant during the radiation-dominated era $(H \sim$ $\left.a^{-2}\right)$. The requirements that the produced dilatons do not overclose the universe $\left(\Omega_{\chi}<1\right)$ in the radiation era thus imposes the condition

$$
H_{1} \lesssim M_{p}
$$

which is also needed from a similar constraint on gravitons (this constraint is valid not only for the particular case (5.1) but also, more generally, for all growing dilaton spectra, whose integration leads to an $\Omega_{\chi}(t)$ similar to that of eq.(5.2)).

If the dilaton would be massless, this would be the end of the story. However, in spite of some recent attempt [54] trying to motivate the possibility of a massless dilaton in a string theory context, present conventional wisdom seems to favor a non-vanishing dilaton mass, with a mass value closely related, in particular, to the phenomenology of supersymmetry breaking (see [55] for a recent discussion). But, even independently from possible supersymmetry motivations, a non-vanishing mass seems to be a compulsory consequence of the fact that dilatons couple nonuniversally to macroscopic matter, with coupling strength larger than (or at least equal to) the gravitational one [4]. We shall reproduce below, for completeness, the argument given in ref. [4] making it actually slightly more general.

The large distance behavior of dilaton couplings is determined indeed by the string effective action $\Gamma$, whose general form, including possible loop corrections, can be written as

$$
\begin{gathered}
\Gamma=-\int d^{D} x \sqrt{|G|}\left[Z_{R}(\phi) R(G)+Z_{\phi}(\phi) G^{\mu \nu} \partial_{\mu} \phi \partial_{\nu} \phi+V(\phi)\right]+\Gamma_{M}, \\
\Gamma_{M}=\sum_{i} \int d^{D} x \sqrt{|G|}\left[\frac{1}{2} Z_{k}^{i}(\phi) G^{\mu \nu} \partial_{\mu} \psi_{i} \partial_{\nu} \psi_{i}+Z_{m}^{i}(\phi) \psi_{i}^{2}+\text { interaction terms }\right]
\end{gathered}
$$

Here $Z_{R}, Z_{\phi}, Z_{k}, Z_{m}$ are complicated (known in principle, but unknown in practice) coupling functions, $G_{\mu \nu}$ is the (dimensional) sigma-model metric, and we have represented the matter part of the action as a set of (dimensionless) scalar fields $\psi_{i}$ (fundamental fermions can be added without difficulty [4]). In order to evaluate 
the effective dilaton couplings we must restore, first of all, the canonical form of the kinetic energy terms, by rescaling field and masses. The dilaton coupling to matter fields is then obtained from the effective interaction Lagrangian, expressed in terms of the rescaled variables.

To this aim we note that the graviton kinetic term of eq.(5.4) reduces to the canonical Einstein Lagrangian by putting

$$
G_{\mu \nu}=g_{\mu \nu} M_{p}^{2} Z_{R}^{-2 /(d-1)}
$$

where $g_{\mu \nu}$ is the dimensionless Einstein metric. By defining a new scalar field (with canonical dimensions) $\sigma$, such that

$$
\frac{d \sigma}{d \phi}=M_{p}^{(d-1) / 2}\left[\frac{2 d}{d-1}\left(\frac{d \ln Z_{R}}{d \phi}\right)^{2}-2 \frac{Z_{\phi}}{Z_{R}}\right]^{1 / 2}
$$

and new rescaled matter fields

$$
\hat{\psi}_{i}=M_{p}^{(d-1) / 2} \sqrt{\frac{Z_{k}^{i}(\sigma)}{Z_{R}(\sigma)}} \psi_{i}
$$

the action (5.4) can be written in canonical form as

$$
\begin{aligned}
\Gamma=-\int d^{D} x \sqrt{|g|}[ & -\frac{R(g)}{16 \pi G_{D}}+\frac{1}{2} g^{\mu \nu} \partial_{\mu} \sigma \partial_{\nu} \sigma-W(\sigma)+ \\
& \left.+\sum_{i} J\left(\frac{1}{2} g^{\mu \nu} \partial_{\mu} \hat{\psi}_{i} \partial_{\nu} \hat{\psi}_{i}+\frac{1}{2} \mu_{i}^{2}(\sigma) \hat{\psi}_{i}^{2}\right)\right]
\end{aligned}
$$

where $16 \pi G_{D}=M_{p}^{1-d}, W=V M_{p}^{d+1} Z_{R}^{-2 /(d-1)}$ and

$$
\mu_{i}^{2}(\sigma)=\frac{2 M_{p}^{2} Z_{m}^{i}(\sigma)}{Z_{k}^{i}(\sigma) Z_{R}^{2 /(d-1)}(\sigma)}
$$

We expand now the effective matter-dilaton interaction Lagrangian, $\mu_{i}^{2} \hat{\psi}_{i}^{2}$, around the value of $\sigma$ which extremizes the dilaton potential (and which can always be assumed to coincide with $\sigma=0$, after a trivial shift). Defining

$$
\frac{1}{2} \mu_{i}^{2}(\sigma) \hat{\psi}_{i}^{2}=\frac{1}{2} m_{i}^{2} \hat{\psi}_{i}^{2}+g_{i} \sigma \hat{\psi}_{i}^{2}+O\left(\sigma^{2}\right)
$$

we can express the rescaled mass of the matter fields as

$$
m_{i}^{2}=\left[\mu_{i}^{2}(\sigma)\right]_{\sigma=0}=2 M_{p}^{2}\left(\frac{Z_{m}^{i}}{Z_{k}^{i} Z_{R}^{2 /(d-1)}}\right)_{\sigma=0}
$$


and the effective matter-dilaton couplings (including loop corrections) as

$$
\begin{gathered}
g_{i}=\frac{1}{2}\left(\frac{d \mu_{i}^{2}}{d \sigma}\right)_{\sigma=0}=\frac{1}{2}\left(\frac{d \phi}{d \sigma} \frac{d \mu_{i}^{2}}{d \phi}\right)_{\sigma=0}= \\
=\frac{m_{i}^{2}}{2 M_{p}^{(d-1) / 2}}\left[\frac{2 d}{d-1}\left(\frac{d \ln Z_{R}}{d \phi}\right)^{2}-2 \frac{Z_{\phi}}{Z_{R}}\right]_{\phi=0}^{-1 / 2}\left[\frac{d}{d \phi} \ln \left(\frac{\mu_{i}^{2}(\phi)}{M_{p}^{2}}\right)\right]_{\phi=0}
\end{gathered}
$$

In the weak coupling regime $Z_{R}=Z_{\phi}=e^{-\phi}=g^{-2}$, where $g$ is the gauge coupling constant of the superstring unification group, and one finds that the effective dilaton coupling strength $g_{i} / m_{i}$ deviates from the standard "gravitational charge" $\sqrt{4 \pi G_{D}} m_{i}$ by the factor

$$
\begin{aligned}
k_{i}=\frac{g_{i}}{m_{i}^{2} \sqrt{4 \pi G_{D}}} & \simeq\left(\frac{d-1}{2}\right)^{1 / 2}\left[g^{2} \frac{\partial}{\partial g^{2}} \ln \left(\frac{\mu_{i}}{M_{p}}\right)^{2}\right]_{\phi=0} \simeq \\
& \simeq 1+\left[\frac{\partial}{\partial \phi} \ln \left(\frac{Z_{m}^{i}}{Z_{k}^{i}}\right)\right]_{\phi=0}
\end{aligned}
$$

where the last equality refers to $d=3$. As already stressed in Ref.[4], eqs.(5.12) and (5.13) clearly show two important phenomenological effects. The first is that string loop contributions violate the universality of the effective dilaton couplings, as the factors $k_{i}$ being different for particles whose mass has different origins; the second is that the dilaton coupling is even stronger than the graviton coupling, as $k_{i} \geq 1$ for all known cases [4] and, in particular, for the confinement-generated component of hadronic masses. Such a conclusion has been recently challenged [54] on the basis of a possible new mechanism forcing eq.(5.13) to give a vanishing (or very small) result. It is difficult for us to understand how such a cancellation can occur for any realistic present value of the dilaton.

The existence of a non-universal scalar force of gravitational strength may be reconciled with the Eotvos-Dicke-Braginski experiments only if its range is finite [56]. By considering the present results obtained from tests of the equivalence principle [5], it follows in particular that the dilaton corrections to low energy Newtonian gravity are only allowed if their range is smaller than about $1 \mathrm{~cm}$, namely for a dilaton mass

$$
m \gtrsim m_{0}=10^{-4} \mathrm{eV}
$$

We now turn to the discussion of bounds following from the energy stored in the dilaton perturbations (or, if we prefer, in actual scalar particles associated with that field). Our discussion follows closely that of ref. [8]. We recall now 
that the expression (5.2) for the dilaton energy density was obtained neglecting the contribution of the rest energy to the proper oscillation frequency $E(t)=$ $\sqrt{(k / a)^{2}+m^{2}}$ (see eq.(3.27)). Therefore, for a massive dilaton, eq.(5.2) is only valid at early enough times such that $\omega_{1}(t)=k_{1} / a>m$ (and thus it is certainly not valid today, in view of the previous bound and of eq.(1.4)). At later times eq. (5.2) is to be corrected to take into account the mass contribution. Let us suppose, first of all, that $m<H_{1}=\omega_{1}\left(t_{1}\right)$, so that eq.(5.2) holds initially, at the beginning of the radiation era, and that $\Omega_{\chi}$ stays dominated by the highest mode $\omega_{1}$ also in the non-relativistic regime. The proper frequency $\omega_{1}$ is red-shifted as the curvature scale decreases in time, and the dominant mode becomes non-relativistic at a scale $H_{n r} \equiv H\left(t_{n r}\right)$ such that

$$
\omega_{1}\left(t_{n r}\right)=H_{1} \frac{a_{1}}{a_{n r}}=m
$$

For $H \lesssim H_{n r}$ the oscillation frequency $E_{1}(t)=\sqrt{\omega_{1}^{2}(t)+m^{2}}$ is dominated by the mass contribution, and the corrected dilaton energy density can be obtained from eq.(5.2) through the rescaling

$$
\Omega_{\chi}(t) \rightarrow \frac{m}{\omega_{1}} \Omega_{\chi}(t) \simeq G m H_{1}\left(\frac{H_{1}}{H}\right)^{2}\left(\frac{a_{1}}{a}\right)^{3} \equiv G \frac{m^{4}}{H^{2}}\left(\frac{a_{n r}}{a}\right)^{3}
$$

(see also [8]). The cosmological bounds on $\Omega_{\chi}$ become then bounds on the dilaton mass, and provide us with a phenomenologically allowed region in the $\left(m, H_{1}\right)$ parameter space.

Consider, first of all, the critical density bound $\Omega_{\chi}<1$. Once that the condition (5.3) is satisfied, the dilaton density $\rho_{\chi}$ is certainly subcritical (and constant with respect to the radiation density $\rho_{\gamma}$ ) for all scales $H>H_{n r}$. When the scale drops below $H_{n r}$, however, the dilaton density becomes non-relativistic according to eq.(5.16), the ratio $\rho_{\chi} / \rho_{\gamma}$ begins to grow in time like the scale factor, and the equality $\rho_{\chi}=\rho_{\gamma}$ may be reached at some initial scale $H_{i} \equiv H\left(t_{i}\right)$. We have now various phenomenological possibilities, depending on the value of $H_{i}$ and $H_{n r}$.

Suppose first that the transition to the non-relativistic regime occurs when the universe is already matter-dominated, namely for $H_{n r} \lesssim H_{2}$ (where $H_{2} \sim 10^{-27} \mathrm{eV}$ is the scale of radiation-matter transition) which means, according to the definition $(5.15)$

$$
m \lesssim \sqrt{H_{1} H_{2}}
$$


In the matter-dominated era, the dilaton energy density (in critical units) (5.16) stays fixed at the value

$$
\Omega_{\chi} \simeq G H_{1}^{2} \frac{m}{\sqrt{H_{1} H_{2}}}
$$

The requirement $\Omega_{\chi}<1$ is thus automatically satisfied, in this case, because of eqs.(5.9), (5.17). The same happens if $H_{n r}>H_{2}$, but the scale $H_{i}$ of dilatonradiation equilibrium belongs to the epoch of matter domination, $H_{i}<H_{2}$. In this case, indeed, $H_{n r}=m^{2} / H_{1}$, and $H_{i}$ is fixed (according to eq. $(5.16)$ ) by

$$
\frac{\rho_{\chi}\left(t_{i}\right)}{\rho_{\gamma}\left(t_{i}\right)}=\frac{\rho_{\chi}\left(t_{n r}\right)}{\rho_{\gamma}\left(t_{n r}\right)} \frac{a_{i}}{a_{n r}} \simeq G H_{1}^{2} \frac{m}{\sqrt{H_{1} H_{2}}}\left(\frac{H_{2}}{H_{i}}\right)^{2 / 3}=1
$$

The condition $H_{i}<H_{2}$, reading now $m \lesssim \sqrt{H_{1} H_{2}}\left(G H_{1}^{2}\right)^{-1}$, again implies $\Omega_{\chi}<1$, where $\Omega_{\chi}$ is the constant dilaton density (5.18).

Dilatons are always subdominant even if $H_{n r}>H_{i}>H_{2}$, but the decay scale $H_{d}$ (1.7) is larger than $H_{i}$, so that dilatons are forced to dissipate their coherent energy density, converting it into radiation before becoming dominant. In this case we have, from eq.(5.16),

$$
H_{i}=H_{n r}\left[\frac{\rho_{\chi}\left(t_{n r}\right)}{\rho_{\gamma}\left(t_{n r}\right)}\right]^{2}=\frac{m^{2} H_{1}^{3}}{M_{p}^{4}}<H_{n r}
$$

and the condition $H_{d}>H_{i}$ reads

$$
m>H_{1}\left(\frac{H_{1}}{M_{p}}\right)^{2}
$$

If, on the contrary, $H_{n r}>H_{i}>H_{2}$ and $H_{i}>H_{d}$, then in order to avoid contradiction with the presently observed density of non-relativistic matter it is necessary, first of all, to impose that dilatons already decayed, $H_{d}>H_{0}$. This gives $m^{3}>M_{p}^{2} H_{0}$ (numerically, $m>100 \mathrm{MeV}$ ). This is not sufficient though and one is left, in this case, with two possible alternatives.

The first is the one in which the reheating temperature $T_{r}$ associated with dilaton decay

$$
T_{r} \simeq \sqrt{M_{p} H_{d}} \simeq\left(\frac{m^{3}}{M_{p}}\right)^{1 / 2}
$$

is smaller than the temperature scale $T_{N} \sim 1 \mathrm{MeV}$ required by nucleosynthesis. This provides

$$
m \lesssim 10^{4} \mathrm{GeV}
$$


In such case we must impose that nucleosynthesis occurred before dilaton dominance, $H_{N} \simeq(1 \mathrm{MeV})^{2} / M_{p}>H_{i}$, and that the entropy $\Delta S$ associated with dilaton decay is small enough, in order not to destroy all light nuclei already formed. The temperature $T_{d}$ of the radiation gas already present at the scale $H_{d}$ is in fact, from eq. (5.20),

$$
T_{d}=T_{i}\left(\frac{a_{i}}{a_{d}}\right) \simeq\left(M_{p} H_{i}\right)^{1 / 2}\left(\frac{H_{d}}{H_{i}}\right)^{2 / 3} \simeq\left(\frac{m^{10}}{M_{p} H_{1}^{3}}\right)^{1 / 6}
$$

The reheating of the radiation gas from $T_{d}$ to $T_{r}$ thus produces an entropy increase

$$
\Delta S \simeq\left(\frac{T_{r}}{T_{d}}\right)^{3} \simeq\left(\frac{H_{1}^{3}}{m M_{p}^{2}}\right)^{1 / 2}
$$

By imposing $\Delta S \lesssim 10$ in order to preserve nucleosynthesis [57] one obtains the bound

$$
m \gtrsim 10^{-2} \frac{H_{1}^{3}}{M_{p}^{2}}
$$

The second alternative corresponds to $T_{r} \gtrsim T_{N}$, i.e. $m \gtrsim 10^{4} \mathrm{GeV}$, and allows then a nucleosynthesis phase subsequent to dilaton decay. In such case, the only phenomenological constraint is possibly imposed by primordial baryogenesis. The maximum tolerable amount of entropy, in order not to wash-out any pre-existing baryon-antibaryon asymmetry, is somewhat model-dependent, but in general $\Delta S \lesssim 10^{5}$ seems to be acceptable [57,58]. This implies the bound

$$
m \gtrsim 10^{-10} \frac{H_{1}^{3}}{M_{p}^{2}}
$$

Note, however, that this last condition may be evaded in the case of low energy baryogenesis and, in particular, in the case of baryogenesis associated with the dilaton decay itself $[55,58]$, occurring at scales not much distant from nucleosynthesis.

The bounds so far considered refer to the case in which the dilaton energy density stays always dominated by the contribution of the highest frequency mode $\omega_{1}$, even in the non-relativistic regime. This is certainly true for the distribution (5.1), with spectral index $\delta=3$, but for a more complete phenomenological discussion let us consider also * the coherent oscillations, with frequency $m$, of the

$*$ The necessity of considering lower modes and of distinguishing two intervals in $\delta$ was pointed out to us by A. Starobinsky. 
lower modes with $\omega\left(t_{m}\right) \lesssim m$, which begin at the scale $H_{m} \equiv H\left(t_{m}\right)=m$, when the mode $\omega_{1}$ is still relativistic $\left(m>H_{n r}\right)$. For $H \lesssim m$ such modes provide a non-relativistic contribution to the dilaton energy density which can be written (for a general spectrum with $\delta>0$ ) as

$$
\Omega_{\chi}(t) \simeq G m^{2}\left(\frac{H_{1}}{H}\right)^{2}\left(\frac{m}{H_{1}}\right)^{\frac{\delta}{2}}\left(\frac{a_{m}}{a}\right)^{3}=G m^{2}\left(\frac{H_{1}}{H}\right)^{2}\left(\frac{m}{H_{1}}\right)^{\frac{\delta-3}{2}}\left(\frac{a_{1}}{a}\right)^{3}
$$

This contribution is initially negligible with respect to the relativistic part of the dilaton energy density, $\sim G H_{1}^{2}$, dominated by $\omega_{1}$. However, during the radiation era it grows in time with respect to $G H_{1}^{2}$, and it may dominate the total dilaton energy if the equality

$$
G m^{2}\left(\frac{H_{1}}{H}\right)^{2}\left(\frac{m}{H_{1}}\right)^{\frac{\delta}{2}}\left(\frac{H}{m}\right)^{3 / 2}=G H_{1}^{2}
$$

occurs at a scale $H>H_{n r}=m^{2} / H_{1}$, namely for $\left(m / H_{1}\right)^{\delta-1}>1$, which implies $\delta<1$.

The previous bounds, obtained in the hypothesis of $\omega_{1}$-dominance, are thus valid for all growing spectra with $\delta \geq 1$. For lower spectral slopes, $0<\delta<1$, the dominant contribution to $\Omega_{\chi}$ (the one to be bounded) becomes that of eq.(5.28) and, as a consequence, the bounds acquire a dependence on $\delta$. Indeed, let $H_{1}$ be the scale marking the equality $\rho_{\chi}=\rho_{\gamma}$. Then the condition $\Omega_{\chi}<1$, with $\Omega_{\chi}$ given by eq.(5.28) and $0<\delta<1$, is always satisfied for $H_{i}<H_{2}$, which means

$$
m<\left(H_{2} M_{p}^{4} H_{1}^{\delta-4}\right)^{1 /(\delta+1)}
$$

It is also satisfied if $H_{i}>H_{2}$, but $H_{d}>H_{i}$, which means

$$
m>\left(H_{1}^{4-\delta} M_{p}^{-2}\right)^{1 /(2-\delta)}
$$

If, however, $H_{i}>H_{2}$ and $H_{i}>H_{d}$, then the dilatons must have already decayed, $H_{d}>H_{0}$. Their decay generates an entropy

$$
\Delta S=\left(\frac{T_{r}}{T_{d}}\right)^{3}=\left(\frac{H_{1}^{4-\delta} m^{\delta-2}}{M_{p}^{2}}\right)^{1 / 2}
$$

If $m<10^{4} \mathrm{GeV}$ the reheating temperature is too low to allow nucleosynthesis: we must impose that nucleosynthesis already occurred, $H_{i}<H_{N}$, and that [57] $\Delta S \lesssim 10$, which means

$$
m \lesssim\left(10^{-2} M_{p}^{-2} H_{1}^{4-\delta}\right)^{1 /(2-\delta)}
$$


If, on the contrary, $m>10^{4} \mathrm{GeV}$, the nucleosynthesis scale is subsequent to dilaton decay, and the only possible constraint $[57,58]$ is $\Delta S \lesssim 10^{-5}$, namely

$$
m \lesssim\left(10^{-10} M_{p}^{-2} H_{1}^{4-\delta}\right)^{1 /(2-\delta)}
$$

This complete the compilation of the phenomenological bounds for a growing dilaton spectra, $\delta>0$, with $m<H_{1}$. Let us consider also, for the sake of completeness, the "heavy dilaton" case, $m>H_{1}$, although this case is very unnatural in a string theory context where $H_{1}$ is expected to be close to the Planck scale.

As discussed in Sec.3, in the radiation phase $\left(H<H_{1}, a \sim \eta\right)$ the dilaton modes $\chi_{k}$ decouple from the other scalar fluctuations, and satisfy the free equation (which includes in general the mass contribution)

$$
\bar{\chi}_{k}^{\prime \prime}+\left(k^{2}+m^{2} a^{2}\right) \bar{\chi}_{k}=0, \quad \bar{\chi}_{k}=a \chi_{k}
$$

For all modes that are relativistic at the beginning of the radiation era $\left(\eta=-\eta_{1}\right)$, namely for $k>m a_{1}$, one then recover the dilaton spectrum (3.32) by matching the pre-big-bang solution (3.30) with the plane wave (3.27), which is solution of eq.(5.35) in the case of negligible mass. If, however,

$$
m>H_{1}=\omega_{1}\left(t_{1}\right)=\frac{k_{1}}{a_{1}}
$$

then the mass term cannot be neglected even in the case of the highest mode $k_{1}$. All modes are non-relativistic already at $\eta=-\eta_{1}$, and satisfy the approximate equation

$$
\bar{\chi}_{k}^{\prime \prime}+m^{2} a^{2} \bar{\chi}_{k}=0
$$

By using the identity $m^{2} a^{2}=m^{2} H_{1}^{2} a_{1}^{4} \eta^{2}$, valid in the radiation era, the general solution of (5.37), for $m H_{1} a_{1}^{2} \eta^{2} \simeq\left(m / H_{1}\right)\left(\eta / \eta_{1}\right)^{2}>1$ can be expressed as

$$
\bar{\chi}=\frac{1}{\sqrt{m H_{1} a_{1}^{2} \eta}}\left(c_{+} e^{-i m H_{1} a_{1}^{2} \eta^{2}}+c_{-} e^{i m H_{1} a_{1}^{2} \eta^{2}}\right)
$$

The matching of this solution with the Bessel solution (3.30) gives then the Bogoliubov coefficient for the case $m>H_{1}$

$$
\left|c_{-}(k)\right|^{2} \simeq \frac{m}{H_{1}}\left(k \eta_{1}\right)^{-2|\nu|}
$$

and the corresponding non-relativistic dilaton spectrum (in critical units)

$$
\Omega_{\chi}(\omega, t) \simeq \frac{G \omega}{H^{2}} \frac{d \rho_{\chi}}{d \omega} \simeq G \frac{m \omega^{3}}{H^{2}}\left|c_{-}\right|^{2} \simeq
$$




$$
\simeq G m^{2}\left(\frac{\omega}{\omega_{1}}\right)^{2-2 \alpha}\left(\frac{H_{1}}{H}\right)^{2}\left(\frac{a_{1}}{a}\right)^{3}
$$

(we recall that $2-2 \alpha=3$ for the particular model of pre-big-bang that we have considered).

For a relic background of massive dilatons with $m>H_{1}$, eq.(5.2) is thus to be replaced by the non-relativistic energy density

$$
\Omega_{\chi}(t)=\int^{\omega_{1}} \frac{d \omega}{\omega} \Omega_{\chi}(\omega, t) \simeq G m^{2}\left(\frac{H_{1}}{H}\right)^{2}\left(\frac{a_{1}}{a}\right)^{3}
$$

One must thus impose the bound

$$
m \lesssim M_{p}
$$

to avoid an over-critical density of massive dilatons at the beginning of the radiation era. In this case there are no further bounds on $m$ since, as a consequence of eq.(5.42), the dilaton energy is dissipated before a possible dominance. Indeed, the initial scale $H_{i}$ corresponding to $\Omega_{\chi}=1$ is, from eq.(5.41),

$$
H_{i}=H_{1}\left(\frac{m}{M_{p}}\right)^{4}
$$

Therefore, $H_{i} / H_{d}=H_{1} m / M_{p}^{2}<1$, just because of eq.(5.42) (unless $H_{1}>M_{p}$, but this is to be excluded to avoid over-critical dominance of other massless particles, such as gravitons, produced by the same background transition).

By collecting all previous phenomenological constraints we obtain a final allowed region in the $\left(m, H_{1}\right)$ plane, which depends on $\delta$ and which is illustrated in Fig. 2 for the three cases $\delta=0, \delta=0.5$ and $\delta=1$. We recall that for large enough spectral slope, $\delta>1$, the bounds become slope-independent and coincide with those of the $\delta=1$ case, which defines then the maximum allowed region for a growing dilaton spectrum (as stressed also in [8]). As clearly shown by the pictures, one of the main effects of a positive spectral index is that light, not yet decayed dilatons become compatible with higher and higher inflation scales as $\delta$ ranges from 0 to 1 . If we take, for instance, $H_{1} \simeq 10^{-5} M_{p}$ as a typical reference value, we find allowed mass windows for a presently dominating background of relic dilatons for all spectra with $\delta \gtrsim 0.1$. For $\delta \gtrsim 1$, moreover, even the $T e V$ mass range, which is supported by some supersymmetry breaking arguments [55], but which lies in the most unfavorable region for the various cosmological constraints, may become compatible with $H_{1} \simeq 10^{-5} M_{p}$. 
We note, finally, that our allowed regions refer to the cosmological amplification of the quantum fluctuations of the dilaton background. The classical dilaton background is assumed here to sit at the minimum of the dilaton potential, with negligible (with respect to quantum fluctuations) oscillations around it. The classical oscillation amplitude, however, could be too large to be negligible. In that case one should add, to the bounds discussed here, the bounds on $m$ obtained by taking into account the contribution of the classical oscillations to the total cosmological energy density [7]. The initial amplitude of such possible classical oscillations depends, however, on the details of the transition from the accelerated to the decelerated regime. Having neglected such possible additional bounds, the allowed region determined here is to be regarded, for each value of $\delta$, as the maximal allowed region in parameter space.

\section{Conclusions.}

In this paper we have presented the general solution of the equations obtained from the low energy string effective action, for the case of space-independent background fields, vanishing dilaton potential and classical strings as possible matter sources. In the perfect fluid approximation, the solution is uniquely fixed by the choice of the equation of state. We have shown that a model of initial sources such as a pressureless gas of weakly interacting strings provides an approximate, but consistent solution to the full system of background equations and string equations of motion.

This model supports a scenario in which an initial flat perturbative string vacuum evolves towards a high curvature, strong coupling regime through a phase of accelerated expansion or contraction. This is the so-called pre-big-bang epoch, originally motivated in $[2,25,27]$ by the duality symmetries of the string effective action. Accelerated contraction, typical of an Einstein frame representation of the pre-big-bang scenario, works as well as the more conventional inflationary kinematic (accelerated expansion) in order to solve the standard cosmological problems.

We have derived, for an isotropic background, the general coupled system of scalar (metric plus dilaton) perturbation equations, and shown that the transition from the pre-big-bang phase to the standard, radiation-dominated cosmology, is 
associated with a copious production of cosmic dilatons, whose spectral distribution grows with frequency. We have discussed the consequent phenomenological bounds on the dilaton mass and on the inflation scale, by combining it with other bounds obtained from tests of the equivalence principle. As a result, we have found allowed windows for the dilaton mass compatible with a possible "dilatonic" solution of the dark matter problem.

The particular model of pre-big-bang considered in this paper leads, however, to scalar metric perturbations which cannot be taken as the origin of the observed CMBR anisotropy, since their spectrum grows too fast with frequency. This can be a welcomed result to people believing in a different source of anisotropy (e.g. in cosmic strings, which could naturally arise in this context as remnants of the violent, non-adiabatic transition from the growing to the decreasing curvature regime). On the other hand, such a result cannot be really taken as typical of our scenario, since we have neglected various effects which may decouple metric and dilaton perturbations leading to a different (possibly flatter) spectrum of metric perturbations. We have in mind, for instance, the contribution of matter sources to the background solutions during the phase of parametric amplification of the vacuum fluctuations and/or a non-trivial evolution of the internal dimensions, whose dynamical compactification introduces at least one additional scalar variables in the perturbations equations.

The most serious omission of this paper is, however, the lack of a detailed description of the transition between the pre-big-bang regime (growing dilaton and curvature scale) and the post-big-bang regime (constant dilaton and decreasing curvature scale). We avoided to face this problem in this paper, as we believe that the low energy effective action adopted here cannot represent any longer an adequate approximation for that purpose. A recent investigation [59] strongly suggests that higher curvature corrections should play a fundamental role in solving the "gracious exit" problem in string cosmology. Furthermore a non-perturbative dilaton potential must be added in the post-big-bang era in order to pin down the dilaton to its present value and to give it a mass.

We do not conceal that the high-curvature transition from inflation to standard cosmology is, at present, the least understood aspect of the whole string cosmology scenario and that it certainly deserves future detailed investigations. We stress however that, once the mechanism which stops the growth of the dilaton and of the curvature and converts non-adiabatically their kinetic energy into radiation is clarified, the phenomenology developed in this paper should remain 
valid without further modifications and quite independently of the details of the transition process.

Acknowledgments. We are grateful to R. Brandenberger, R. Brustein, M. Giovannini, K. Meissner, V. Mukhanov, R. Ricci and A. Starobinski for many helpful discussions. G. V. wishes to thank A. Linde for a useful correspondence. M. G. wishes to thank the CERN Theory Division for hospitality and financial support during part of this work. 


\section{References}

1. L. P. Grishchuk, Sov. Phys. JEPT 40, 409 (1975);

A. A. Starobinski, JEPT Lett. 30, 682 (1979);

V. A. Rubakov, M. Sazhin and A. Veryaskin, Phys. Lett. B115, 189 (1982);

R. Fabbri and M. Pollock, Phys. Lett. B125, 445 (1983);

L. Abbott and M. Wise, Nucl. Phys. B244, 541 (1984)

2. M. Gasperini and G. Veneziano, Astropart. Phys. 1, 317 (1993)

3. M. Gasperini and M. Giovannini, Phys. Rev. D47, 1529 (1993)

4. T. R. Taylor and G. Veneziano, Phys. Lett. B213, 459 (1988);

5. See for instance E. Fischbach and C. Talmadge, Nature 356, 207 (1992)

6. J. Ellis et al., Phys. Lett. B228, 264 (1989).

7. G. D. Coughlan et al., Phys. Lett. B131, 59 (1983);

J. Ellis, D. V. Nanopoulos and M. Quiros, Phys. Lett. B174,176 (1986);

J. Ellis, N. C. Tsamish and M. Voloshin, Phys. Lett. B184, 291 (1986);

B. De Carlos et al., Phys. Lett. B318, 447 (1993)

8. M. Gasperini, Relaxed bound on the dilaton mass in a string cosmology scenario, DFTT-03/94 (January 1994), gr-qc/9401026

9. A. S. Goncharov, A. D. Linde and M I. Vysotsky, Phys. Lett. B147, 279 (1984)

10. G. Smoot et al., Astrophys. J. 396, L1 (1992)

11. C. L. Bennet et al., Cosmic temperature fluctuations from two years of COBE DMR observations, astro-ph/9401012 (January 1994)

12. T. Piran et al., Limits on the primordial fluctuation spectrum, void sizes and CMBR anisotropy, astro-ph/9305019

13. A. D. Linde, Phys. Lett. B259, 38 (1991);

Phys. Rev. D49 (1994) (in press)

14. E. J. Copeland et al., False vacuum inflation with Einstein gravity, SUSSEXAST 94/1-1 (January 1994), astro-ph/9401011

15. S. Mollerach, S. Matarrese and F. Lucchin, Blue perturbation spectra from inflation, astro-ph/9309054

16. J. Mc Donald, Phys. Rev. D48, 2468 (1993)

17. E. W. Kolb, D. Salopek and M. S. Turner, Phys. Rev. D42, 3925 (1990)

18. M. S. Turner and L. M. Widrow, Phys. Rev. D37, 3428 (1988)

19. C. Lovelace, Phys. Lett. B135, 75 (1984);

E. S. Fradkin and A. A. Tseytlin, Nucl. Phys. B261, 1 (1985); 
C. G. Callan, D. Friedan, E. J. Martinec and M. J. Perry, Nucl. Phys. B262, $593(1985)$

20. M. Gasperini and G. Veneziano, Phys. Lett. B277, 256 (1992)

21. K. A. Meissner and G. Veneziano, Phys. Lett. B267, 33 (1991)

22. K. A. Meissner and G. Veneziano, Mod. Phys. Lett. A6, 3397 (1991)

23. M. Gasperini and G. Veneziano, Mod. Phys. Lett. A8, 3701 (1993)

24. M.Mueller, Nucl. Phys. B337, 37 (1990)

25. G. Veneziano, Phys. Lett. B265, 287 (1991)

26. A. A. Tseytlin, Mod. Phys. Lett. A6, 1721 (1991)

27. M. Gasperini, N. Sanchez and G. Veneziano, Nucl. Phys. B364, 365 (1991); Int. J. Mod. Phys. A6, 3853 (1991)

28. J. Bardeen, Phys. Rev. D22, 1822 (1980)

29. M. Sasaki, Prog. Theor. Phys. 70, 394 (1983)

30. V. Mukhanov, H. A. Feldman and R. Brandenberger, Phys. Rep. 215, 203 (1992)

31. S. Mollerach and S. Matarrese, Phys. Rev. D45, 1961 (1992)

32. N. Deruelle, C. Gundlach and D. Langlois, Phys. Rev. D46, 5337 (1992)

33. N. Deruelle, C. Gundlach and D. Polarski, Class. Quantum Grav. 9, 137 (1992)

34. L. P. Grishchuk, in Proc. of the 6th Marcel Grossmann Meeting (Kyoto, 1991), ed. by H. Sato (World Scientific, Singapore, 1992)

35. M. Sasaki, Prog. Theor. Phys. 76, 1036 (1986)

36. V. F. Mukhanov, Sov. Phys. JEPT 67, 1297 (1988)

37. E. D. Stewart and D. H. Lyth, Phys. Lett. B302, 171 (1993)

38. V. N. Lukash, Sov. Phys. JEPT 52, 807 (1980)

39. G. V. Chibisov and V. N. Mukhanov, Mon. Not. R. Astron. Soc. 200, 535 (1982)

40. N. Deruelle, C. Gundlach and D. Langlois, Phys. Rev. D45, R3301 (1992)

41. M. Gasperini and G. Veneziano, in preparation

42. N. D. Birrel and P. C. W. Davies, Quantum fields in curved space (Cambridge University Press, Cambridge, England, 1982)

43. F. Lucchin and S. Matarrese, Phys. Lett. B164, 282 (1985);

B. Allen, Phys. Rev. D37, 2078 (1988);

V. Sahni, Phys. Rev. D42, 435 (1990);

L. P. Grishchuk and M. Solokhin, Phys. Rev. D43, 2566 (1991);

M. Gasperini and M. Giovannini, Phys. Lett. B282, 36 (1992) 
44. L. P. Grishchuk and Y. V. Sidorov, Phys. Rev. D42, 3413 (1990);

L. P. Grishchuk, Phys. Rev. Lett. 70, 2371 (1993)

45. M. Gasperini and M. Giovannini, Phys. Lett. B301, 334 (1993)

46. M. Gasperini, M. Giovannini and G. Veneziano, Phys. Rev. D48, R439 (1993)

47. R. Brandenberger, V. Mukhanov and T. Prokopec, Phys. Rev. Lett. 69, 3606 (1992);

Phys. Rev. D48, 2443 (1993)

48. S. W. Hawking, Phys. Lett. B115, 295 (1982);

A. Linde, Phys. Lett. B116, 335 (1982);

A. A. Starobinski, Phys. Lett. B117, 175 (1982);

A. Guth and S. Y. Pi, Phys. Rev. Lett. 49, 1110 (1982);

J. M. Bardeen, P. S. Steinhardt and M. S. Turner, Phys. Rev. D28, 679 (1983)

49. G. Veneziano, Europhys. Lett. 2, 133 (1986)

50. A. Guth, Phys. Rev. D23, 347 (1981)

51. M. Gasperini, M. Giovannini, K. A. Meissner and G. Veneziano, in preparation

52. F. Lucchin and S. Matarrese, Ref. 43

53. Y. Hu, M. S. Turner and E. J. Weinberg, Inflation as the unique solution to the horizon and flatness problem, Fermilab-Pub-92/363-A, astro-ph/9302002

54. T. Damour and A. M. Polyakov, The string dilaton and a least coupling principle, IHES/P/94/1 (January 1994), hep-th/9401069

55. T. Banks, D. V. Kaplan and A. Nelson, Cosmological implications of dynamical supersymmetry breaking, UCSD/PTH 93-26, RU-37, hep-ph/9308292

56. M. Gasperini, Gen. Rel. Grav. 16, 1031 (1984)

57. J. Ellis, D. V. Nanopoulos and M. Quiros, Ref. 7

58. B. De Carlos et al., Ref.7

59. R. Brustein and G. Veneziano, The graceful exit problem in string cosmology, CERN-TH.7179/94 (February 1994) 


\section{Figure captions}

Fig.1 The dashed area defines the allowed windows for the dilaton mass $m$ (given in units of $m_{0}=10^{-4} \mathrm{eV}$ ) and the final inflation scale $H_{1}$ which are compatible with a present large contribution of non-relativistic dilatons to $\Omega$, under the assumption that they are produced with a fast enough growing spectrum, $\delta \geq$ 1. For lower spectral slopes the allowed window is shifted towards lower values of mass, according to eq.(1.9). Masses higher than $100 \mathrm{MeV}$ are excluded by dilaton decay, masses lower than $10^{-4} \mathrm{eV}$ by tests of the equivalence principle. Inflation scales higher than $M_{p}$ are excluded in order to avoid over-critical density in the primordial relativistic particle production.

Fig.2 Maximum allowed region (inside the full lines) relative to the cosmological production of dilatons with growing spectrum, illustrated for three different spectral slopes $\delta=0, \delta=0.5$ and $\delta=1$ (the last case applies to all $\delta \geq 1)$. The dilaton mass is given in units of $m_{0}=10^{-4} \mathrm{eV}$. The lines marked by $a, b, c, d, e, f, g$ represent the most significant bounds quoted in the text, and correspond respectively to: a) $m=m_{0}$, lower bound on $m$ from the equivalence principle; b) $H_{1}=M_{p}$, upper bound on $H_{1}$ from the closure density; c) $T_{r}=1 \mathrm{MeV}$, lower bound on the reheating temperature for nucleosynthesis; d) $m=M_{p}$, upper bound on $m>H_{1}$ from the closure density; e) $m=\left(H_{2} M_{p}^{4} H_{1}^{\delta-4}\right)^{1 /(\delta+1)}$, upper bound on $m$ from the present matter-to-radiation energy density ratio; f) $m=\left(10^{-10} M_{p}^{-2} H_{1}^{4-\delta}\right)^{1 /(2-\delta)}$, upper limit on entropy production in dilaton decay from primordial baryogenesis; g) $m=\left(10^{-2} M_{p}^{-2} H_{1}^{4-\delta}\right)^{1 /(2-\delta)}$, upper limit on entropy production from nucleosynthesis. 
This figure "fig1-1.png" is available in "png" format from: http://arXiv.org/ps/gr-qc/9403031v1 
This figure "fig1-2.png" is available in "png" format from: http://arXiv.org/ps/gr-qc/9403031v1 